\title{
PROBABILITY AND EXPECTATION INEQUALITIES
}

by

\section{Stamatis Cambanis* and Gordon Simons**} University of North Carolina

\begin{abstract}
This paper introduces a mathematical framework within which a wide variety of known and new inequalities can be viewed from a common perspective. Probability and expectation inequalities of the following types are considered: (a) $P(Z \in A) \geq P\left(Z^{\prime} \in A\right)$ for some class of sets $A$, (b) $E k(Z) \geq E k\left(Z^{\prime}\right)$ for some class of functions $k$, and (c) $E l(Z) \geq E k\left(Z^{\prime}\right)$ for some class of pairs of functions $l$ and $k$. It is shown, sometimes using explicit constructions of $Z$ and $Z^{\prime}$, that, in several cases, (a) $\Longleftrightarrow(b) \Leftrightarrow(c)$; included here are cases of normal and elliptically contoured distributions. A case where (a) $\Rightarrow$ (b) $\Leftrightarrow$ (c) is studied and is expressed in terms of "n-monotone" functions for (some of) which integral representations are obtained. Also, necessary and sufficient conditions for (c) are given.
\end{abstract}

Key Words and Phrases: probability inequalities, expectation inequalities, normal distribution, elliptically contoured distributions, stochastic orderings, quasi-monotone functions, n-monotone functions

\footnotetext{
* Research supported by the Air Force Office of Scientific Research under Grant AFOSR-75-2796.

** Research supported by the National Science Foundation under Grant MCS78-01240.
} 


\section{Introduction}

There is an extensive literature dealing with probability inequalities of the form

$$
P(Z \in A) \geq P\left(Z^{\prime} \in A\right), \quad A \in A \text {, }
$$

and expectation inequalities of the form

$$
\operatorname{Ek}(Z) \geq \operatorname{Ek}\left(Z^{\prime}\right), \quad k \in F_{1},
$$

where $Z$ and $Z^{\prime}$ are random vectors (or more general random elements) with common range space $R, A$ is a class of (Borel) subsets of $R$, and $F_{1}$ is a class of real (measurable) functions on $R$. Here we also focus attention on expectation inequalities of the form

$$
E l(Z) \geq E k\left(Z^{\prime}\right), \quad(l, k) \in F_{2},
$$

where $F_{2}$ is a class of pairs of (measurable) functions on $R$. When the classes $A, F_{1}, F_{2}$ are progressively richer then conditions $(1.1),(1.2),(1.3)$ are progressively stronger. Specifically if $1_{A} \in F_{1}$, i.e. if $1_{A} \in F_{1}$ for all $A \in A$, then $(1.1) \Leftarrow(1.2) ;$ and if $F_{1} \subset\left\{k:(k, k) \in F_{2}\right\}=: F_{21}$ then $(1.2) \Leftarrow(1.3)$. The more interesting conclusions are therefore those which lead from (1.1) to (1.2) to $(1.3)$

The first question of interest is of course to describe conditions on the distributions of $Z$ and $Z$ ' which guarantee (1.1) for specific classes $A$ of sets, and there is a vast literature on this. The second question is, given a class of sets $A$, to describe a class of functions $F_{1}$, depending of course on $A$, for which $(1.1) \Rightarrow(1.2)$; if such a class $F_{1}$ contains $1_{A}$ then in fact $(1.1) \Leftrightarrow(1.2)$. The third question is, given a class of functions $F_{1}$, to describe a class $F_{2}$ of 
pairs of functions for which $(1.2) \Rightarrow(1.3)$; and if furthermore $F_{1} \subset F_{21}$ then $(1.2) \Leftrightarrow(1.3)$. Clearly this equivalence holds for the class $F_{2}$ defined by what may be called the "separation approach":

$$
\begin{aligned}
F_{2}= & \left\{(l, k): \ell \leq \mathrm{m} \leq \mathrm{k} \text { for some } \mathrm{m} \in F_{1},\right. \\
& \text { and the expectations in }(1.3) \text { are defined }\} .
\end{aligned}
$$

(This approach is most useful when there is a simple direct description of $F_{2}$, one that does not predicate the existence of quantities with certain properties.) When positive answers to the second and third questions are feasible, one of the following relationships will follow:

$$
\begin{aligned}
& \text { i. }(1.1) \Rightarrow(1.2) \Rightarrow(1.3) \\
& \text { ii. }(1.1) \Rightarrow(1.2) \Leftrightarrow(1.3) \\
& \text { iii. }(1.1) \Leftrightarrow(1.2) \Leftrightarrow(1.3) .
\end{aligned}
$$

An interesting example of (1.5.iii) is described by Kemperman [5] . Suppose that $R$ is a partially ordered space and that (1.1) holds for the class $A$ of all measurable increasing sets $A$ (i.e. $a \in A$ and $a \leq b$, in the sense of the partial ordering, imply $\mathrm{b} \in \mathrm{A}$ ). Then, by considering simple function approximations, one obtains $(1.1) \Leftrightarrow(1.2)$ where $F_{1}$ is the class of all measurable increasing functions $k$ (in the sense of the partial ordering) for which the expectations in (1.2) are defined. Using the separation approach one also obtains $(1.2) \Leftrightarrow(1.3)$, where $F_{2}$ is defined by $(1.4)$ and has the alternative direct description as the class of all pairs of functions $(\ell, k)$ satisfying

$$
k(x) \leq \ell(y), \quad x \leq y,
$$


and for which the expectations in (1.3) are defined, provided the "separating" increasing function $m$ defined for instance by

$$
m(y)=\sup \{k(x), x \leq y\}
$$

is measurable, as is the case when $R$ is the real line. (We infer from a comment made by Kemperman [5] that measurability of m may fail even in $\mathbb{R}^{2}$.) New examples of (1.5.iii) are described in Section 3 for bivariate random variables with normal distributions (Theorem 3.1) and with certain elliptically contoured distributions (Theorem 3.2).

An interesting example of (1.5.ii) is described in Section 2 when the range of $Z=(X, Y)$ and $Z^{\prime}=\left(X^{\prime}, Y^{\prime}\right)$ is the real plane. If $A$ is the class of all closed symmetric rectangles then (1.1) implies that

$$
E h\left(X^{2}+Y^{2}\right) \geq E h\left(X{ }^{2}+Y^{\prime 2}\right)
$$

for all nonincreasing, convex functions $h$ on the positive half line; i.e. $(1.1) \Rightarrow(1.2)$ where $F_{1}$ is the class of all functions $k(x, y)$ of the form $h\left(x^{2}+y^{2}\right)$ with $h$ as above. It should be pointed out that (1.1) no longer implies (1.2) if $h$ is either not nonincreasing or not convex; convexity would be unnecessary if $(1.1)$ implied that $x^{2}+y^{2}$ is stochastically larger than $\mathrm{X}^{\prime}+\mathrm{Y}^{2}$, which is not true in general. In order to use the separation approach, we note that functions $f$ and $g$ on the positive real line can be separated by a convex function $h$,

$$
\mathrm{f} \leq \mathrm{h} \leq \mathrm{g},
$$

if and only if

$$
f[\lambda s+(1-\lambda) t] \leq \lambda g(s)+(1-\lambda) g(t), \quad s \leq t, \quad 0 \leq \lambda \leq 1,
$$


and then the convex separating function $h$ can be defined (not necessarily unique1y) by

$$
h(u)=\inf \left\{\frac{t-u}{t-s} g(s)+\frac{u-s}{t-s} g(t), \quad s<u \leq t\right\} .
$$

Also, this choice of $h$, or some simple modification of it, is nonincreasing if and only if

$$
f(t) \leq g(s), \quad s \leq t
$$

Consequent1y (1.1) implies

$$
E g\left(X^{2}+Y^{2}\right) \geq E f\left(X,{ }^{2}+Y^{2}\right)
$$

for all functions $f$ and $g$ satisfying (1.9) and (1.11) and such that the expectations in (1.12) are defined; i.e. (1.1) $\Rightarrow(1.2) \Leftrightarrow(1.3)$ where $F_{2}$ is the class of all pairs of functions $\ell(x, y)=g\left(x^{2}+y^{2}\right), k(x, y)=f\left(x^{2}+y^{2}\right)$ where $f$ and $g$ are described above. Again, in this case, the class $F_{2}$, originally introduced via the separation approach, has a direct description. Section 2 includes additional implications of the form $(1.1) \Rightarrow(1.2)$, which are described for $n$-dimensional vectors $Z$ and $Z^{\prime}(n \geq 2)$, and also integral representations for certain "n-monotone" functions which may be of independent interest.

Kemperman [5] also describes an alternative approach based on a theorem of Strassen [10] which guarantees that when $R$ is a partially ordered complete separable metric space and $A$ is the class of all measurable increasing sets, then (1.1) is equivalent to the following:

There exist two random variables $z_{0}, z_{0}^{\prime}$ with the same marginal distributions as $Z, Z^{\prime}$ and such that $Z_{0} \geq Z_{0}^{\prime}$ a.s. 
It is then immediate that $(1.13) \Rightarrow(1.3) \Rightarrow(1.2) \Rightarrow(1.1)$ where $F_{1}, F_{2}$ are defined in the paragraph describing Kemperman's example, and thus $(1.13) \Leftrightarrow(1.1) \Leftrightarrow(1.2) \Leftrightarrow(1.3)$. It turns out that this use of surrogate random variables with certain specified a.s. properties (cf. (1.13)), the "surrogate approach," provides a necessary and sufficient condition for expectation inequalities of the type (1.3) in cases where no other approach seems to work (including the separation approach) and even in cases where no useful necessary and sufficient condition for (1.3) of the type (1.1) can be found. We illustrate the usage of this surrogate approach in a case treated in Section 3 .

Let $Z=(X, Y)$ and $Z^{\prime}=\left(X^{\prime}, Y^{\prime}\right)$ be two-dimensional random vectors and $A$ the class of all principal lower and upper ideals in $\mathbb{R}^{2}$, i.e. all rectangles of the forms $(-\infty, x] \times(-\infty, y]$ and $[x, \infty) \times[y, \infty)$. Then $(1.1)$ is equivalent to saying that $Z$ and $Z^{\prime}$ have common marginal distributions and that (1.1) holds for all principal lower ideals $(-\infty, x] \times(-\infty, y]$. It is shown in [2] and [12] that $(1.1) \Leftrightarrow(1.2)$ where $F_{1}$ is the class of all quasi-monotone functions $k$, i.e. functions $\mathrm{k}$ which satisfy the inequalities

$$
k\left(x_{1}, y_{1}\right)+k\left(x_{2}, y_{2}\right) \geq k\left(x_{1}, y_{2}\right)+k\left(x_{2}, y_{1}\right), \quad x_{1} \leq x_{2}, \quad y_{1} \leq y_{2},
$$

for which the expectations in (1.2) are defined and which satisfy some minor regularity conditions. It is not completely clear how the quasi-monotonicity condition (1.14) should be modified in order to derive inequalities of type (1.3). The separation approach would yield (1.5.iii) with $F_{2}$ defined by (1.4) as the class of all pairs of functions $l, k$ which are separated by a quasi-monotone function. It then follows that

$$
\ell\left(x_{1}, y_{1}\right)+\ell\left(x_{2}, y_{2}\right) \geq k\left(x_{1}, y_{2}\right)+k\left(x_{2}, y_{1}\right), \quad x_{1} \leq x_{2}, \quad y_{1} \leq y_{2},
$$


but we have been unable to find a direct description of the class $F_{2}$ defined through the separation approach. Condition (1.15) is necessary but not sufficient for $\ell, k$ to be separated by a quasi-monotone function, as shown by an example in Section 3. Another example shows that (1.1) does not imply (1.3) for all functions $k$ and \& satisfying (1.15) (and for which the expectations in (1.3) are defined). One could conceivably require $(l, k) \in F_{2}$ to satisfy additional inequalities which are in the same spirit as (1.15). For instance, if the additional inequalities

$$
\begin{array}{r}
\ell\left(x_{1}, y_{1}\right)+\ell\left(x_{2}, y_{3}\right)+\ell\left(y_{3}, x_{2}\right) \geq k\left(x_{1}, y_{3}\right)+k\left(x_{2}, y_{2}\right)+k\left(x_{3}, y_{1}\right), \\
x_{1} \leq x_{2} \leq x_{3}, \quad y_{1} \leq y_{2} \leq y_{3},
\end{array}
$$

do not hold, it is possible to construct examples where (1.1) holds but (1.3) fails. But even these inequalities are insufficient and we have failed to obtain usable conditions describing the class $F_{2}$ by continuing with this approach. An alternative approach is to assume that $k$ and $\ell$ satisfy no more than (1.15), i.e. to define $F_{2}$ as the class of al1 pairs of functions $k$ and $\ell$ which satisfy (1.15), and for which the expectations in (1.3) are defined, and to impose additional assumptions on $Z$ and $Z^{\prime}$, i.e. to strengthen condition (1.1). This can be best achieved through a variation of the surrogate approach. To this end consider the following condition:

(CO) There exists a four-dimensional random vector $\left(X_{1}, X_{2}, Y_{1}, Y_{2}\right)$ whose values are in the set $F=\left\{\left(x_{1}, x_{2}, y_{1}, y_{2}\right)=\left(x_{2}-x_{1}\right)\left(y_{2}-y_{1}\right) \geq 0\right\}$ and whose bivariate marginals $\mathrm{F}_{11}, \mathrm{~F}_{12}, \mathrm{~F}_{21}, \mathrm{~F}_{22}$ for $\left(X_{1}, Y_{1}\right),\left(X_{1}, Y_{2}\right),\left(X_{2}, Y_{1}\right),\left(X_{2}, Y_{2}\right)$ respectively satisfy $\mathrm{F}_{11}+\mathrm{F}_{22}=2 \mathrm{H}$ and $\mathrm{F}_{12}+\mathrm{F}_{21}=2 \mathrm{H}^{\prime}$, where $\mathrm{H}$ and $\mathrm{H}^{\prime}$ are the distribution functions of $Z$ and $Z$ ' respectively.

When (1.15) holds, condition (CO) implies 


$$
\ell\left(X_{1}, Y_{1}\right)+\ell\left(X_{2}, Y_{2}\right) \geq k\left(X_{1}, Y_{2}\right)+k\left(Y_{2}, X_{1}\right) \text { a.s. }
$$

which, upon taking expectations (assuming they are defined), yields (1.3). Hence $(C O) \Rightarrow(1.3)$. It is shown in Section 3 that when $Z$ and $Z^{\prime}$ are normally distributed with common means and variances then

$$
\rho \geq \rho^{\prime} \Leftrightarrow(\mathrm{CQ}) \Leftrightarrow(1.1) \Leftrightarrow(1.2) \Leftrightarrow(1.3),
$$

where $\rho\left(\rho^{\prime}\right)$ denotes the correlation between the components of $Z\left(Z^{\prime}\right)$. In Section 3 , a similar result is obtained when $Z$ and $Z^{\prime}$ have elliptically contoured distributions; and also a generalization from two to higher dimensions is described.

For the example of the preceding paragraph, as was mentioned, (1.1) does not imply (1.3) in general. It is shown in Section 4 that (CO) $\Leftrightarrow(1.1)^{\prime} \Leftrightarrow(1.3)$, where $A_{2}$ is the class of all pairs of Borel sets $A$ and $A^{\prime}$ in the plane which are such that the functions $1_{A}$ and $1_{A}$, satisfy (1.15) and

$$
P(Z \in A) \geq P\left(Z^{\prime} \in A^{\prime}\right), \quad\left(A, A^{\prime}\right) \in A_{2}
$$

Using a generalization of a theorem by Strassen [10] we obtain in Section 4 several further results of the type (CO) $\Leftrightarrow(1.1)^{\prime} \Leftrightarrow(1.3)$, where (1.1)' is stronger than (1.1). Among these the following is related to the inequalities of Section 2 described earlier. When $R=R^{2}$, $A$ is the class of all closed symmetric rectangles; $F_{1}$ is the class of all functions $k$ which satisfy

$$
k\left(x_{1}, y_{1}\right) \leq k\left(x_{2}, y_{2}\right), \quad\left|x_{2}\right| \leq\left|x_{1}\right| \text { or }\left|y_{2}\right| \leq\left|y_{1}\right| \text {, }
$$


and for which the expectations in (1.2) are defined; and $F_{2}$ is the class of all pairs of functions $\ell$, and $k$ which satisfy

$$
\ell\left(x_{2}, y_{2}\right) \geq k\left(x_{1}, y_{1}\right), \quad\left|x_{2}\right| \leq\left|x_{1}\right| \text { or }\left|y_{2}\right| \leq\left|y_{1}\right|
$$

and for which the expectations in (1.3) are defined; it is shown in Section 4 that $(1.1) \Longleftrightarrow(1.2) \Leftrightarrow(1.3)$. It should be noted that the functions $k(x, y)=f\left(x^{2}+y^{2}\right)$, with $f$ nonincreasing and convex, considered in Section 2 , do not belong to the class $F_{1}$. Finally Section 4 derives several new inequalities of the type (1.1) and (1.3) for normal and elliptically contoured distributions.

\section{2. n-monotone functions}

In this section we develop inequalities for expectations of n-monotone functions (to be defined below) of the squares of the moduli of n-dimensional random vectors. We begin with the case $n=2$ (Theorem 2.1 ) and then proceed to the general case $n \geq 2$ (Theorem 2.2). In the process of establishing Theorem 2.2 we develop an integral representation for certain n-monotone functions (Lemma 2.3) which may be of independent interest.

Theorem 2.1. Suppose $\mathrm{Z}=(\mathrm{X}, \mathrm{Y})$ and $\mathrm{Z}^{\prime}=\left(\mathrm{X}^{\prime}, \mathrm{Y}^{\prime}\right)$ are bivariate random vectors for which

$$
P(|X| \leq a,|Y| \leq b) \geq P\left(\left|X^{\prime}\right| \leq a,\left|Y^{\prime}\right| \leq b\right), \quad a \geq 0, \quad b \geq 0 .
$$

Then

$$
E f\left(X^{2}+Y^{2}\right) \geq E f\left(X{ }^{2}+Y^{\prime 2}\right)
$$

for every nonincreasing convex function $f$ on $[0, \infty)$. 
Proof. Condition (2.1) is equivalent to saying that $\mathrm{ax}^{\prime}{ }^{2} \vee \mathrm{bY}^{\prime}{ }^{2}$ is stochastically larger than $a X^{2} \vee b Y^{2}$ for $a \geq 0, b \geq 0$, where $u \vee v$ denotes the maximum of $u$ and $v$. Thus for any bounded nonincreasing function $h$ on $[0, \infty)$,

$$
\operatorname{Eh}\left(a X^{2} \vee b Y^{2}\right) \geq E h\left(a X,^{2} v b Y !^{2}\right), \quad a \geq 0, \quad b \geq 0
$$

and, consequently,

$$
E \int_{0}^{\frac{\pi}{2}} h\left(\frac{\mathrm{X}^{2}}{\cos ^{2} \theta} \vee \frac{\mathrm{Y}^{2}}{\sin ^{2} \theta}\right) \sin \theta \cos \theta d \theta \geq E \int_{0}^{\frac{\pi}{2}} \mathrm{~h}\left(\frac{\mathrm{X}^{2}}{\cos ^{2} \theta} \vee \frac{\mathrm{Y}^{2}}{\sin ^{2} \theta}\right) \sin \theta \cos \theta \mathrm{d} \theta \text {. }
$$

Now with the substitution of $\left(x^{2}+y^{2}\right)$ u for $\frac{x^{2}}{\cos ^{2} \theta} v \frac{y^{2}}{\sin ^{2} \theta}$, the integral $\int_{0}^{\frac{\pi}{2}} h\left(\frac{x^{2}}{\cos ^{2} \theta} \times \frac{y^{2}}{\sin ^{2} \theta}\right) \sin \theta \cos \theta$ d $\theta$ simplifies to

$$
\frac{1}{2} \int_{1}^{\infty} \frac{h\left(\left(x^{2}+y^{2}\right) u\right)}{u^{2}} d u
$$

Thus (2.2) holds for functions $f$ of the form $f(s)=\int_{1}^{\infty} \frac{h(s u)}{u^{2}} d u, s \geq 0$. But, according to the lemma below, the class of such functions coincides with the class of bounded nonincreasing convex functions. The unwanted restriction of boundedness is easily removed by truncation: If $f$ is any nonincreasing convex function, then $f \vee(-n)$ is a bounded nonincreasing convex function whose limit, as $\mathrm{n} \rightarrow \infty$, is $\mathrm{f}$. Then (2.2) follows by means of the monotone convergence theorem.

Lemma 2.1. The class of bounded noninereasing convex functions on $[0, \infty)$ and the class of functions $f$ of the form $f(s)=\int_{1}^{\infty} \frac{h(s u)}{u^{2}} d u, s \geq 0$, with $h$ nonincreasing and bounded on $[0, \infty)$, coincide. 
Proof. Suppose $f(s)=\int_{1}^{\infty} \frac{h(s u)}{u^{2}} d u, s \geq 0$, with $h$ nonincreasing and bounded on $[0, \infty)$. Quite obviously $f$ is nonincreasing and bounded. To see that $f$ is convex, observe that $f(s)=s \int_{s}^{\infty} \frac{h(v)}{v^{2}} d v$ for $s>0$ and $f(0)=h(0)$. Thus for $0<s<t$,

$$
\begin{aligned}
f(s)+f(t)-2 f\left(\frac{s+t}{2}\right)= & s \int_{s}^{\infty} \frac{h(v)-h\left(\frac{s+t}{2}\right)}{v^{2}} d v+t \int_{t}^{\infty} \frac{h(v)-h\left(\frac{s+t}{2}\right)}{v^{2}} d v \\
-(s+t) \int_{s+t}^{\infty} \frac{h(v)-h\left(\frac{s+t}{2}\right)}{2} d v & v^{2} \\
& =s \frac{s+t}{\int_{s}^{2}} \frac{h(v)-h\left(\frac{s+t}{2}\right)}{v^{2}} d v+t \int_{\frac{s+t}{2}}^{t} \frac{h\left(\frac{s+t}{2}\right)-h(v)}{v^{2}} d v \geq 0 .
\end{aligned}
$$

The argument when $s=0$ is similar. Consequently, $f$ is convex on $[0, \infty)$. Conversely, suppose $f$ is a bounded nonincreasing convex function on $[0, \infty)$. Define a nonincreasing function $h$ on $[0, \infty)$ as follows:

$$
\begin{aligned}
h(s) & =f(s)-s f^{0}(s) & & \text { for } s>0, \\
& =f(0) & & \text { for } s=0,
\end{aligned}
$$

where $f^{0}(s)$ denotes, for definiteness, the smallest slope among all tangent lines to $f$ at $s$. (Thus, when $f$ is differentiable at $s>0, f^{0}(s)=f^{\prime}(s)$.) Then for fixed $n \geq 1$ and $s>0$,

$$
f(s)-s \frac{f\left(s\left(1+\frac{1}{n}\right)\right)-f(s)}{s / n} \leq h(s) \leq f(s)-s \frac{f(s)-f\left(s\left(1-\frac{1}{n}\right)\right)}{s / n},
$$

due to the convexity of $f$. Thus 


$$
(n+1) f(s)-n f\left(s\left(1+\frac{1}{n}\right)\right) \leq h(s) \leq n f\left(s\left(1-\frac{1}{n}\right)\right)-(n-1) f(s) \text {. }
$$

But

$$
\int_{1}^{\infty} \frac{(n+1) f(s u)-n f\left(\operatorname{su}\left(1+\frac{1}{n}\right)\right)}{u^{2}} d u=(n+1) \int_{1}^{1+\frac{1}{n}} \frac{f(s u)}{u^{2}} d u \rightarrow f(s) \text { as } n \rightarrow \infty \text {, }
$$

since $f$ is continuous at every point $s>0$, and likewise

$$
\int_{1}^{\infty} \frac{n f\left(\operatorname{su}\left(1-\frac{1}{n}\right)\right)-(n-1) f(s u)}{u^{2}} d u \rightarrow f(s) \text { as } n \rightarrow \infty
$$

from which it follows that

$$
f(s)=\int_{1}^{\infty} \frac{h(s u)}{u^{2}} d u, \quad s \geq 0
$$

From this integral it is easily checked that $h$ is bounded.

There are unbounded nonincreasing convex functions $f$ which cannot be expressed in the above integral form, with $h$ nonincreasing and necessarily unbounded, e.g., $f(s)=-s, s \geq 0$. An analogue of Lemma 2.1 can be established for nonincreasing convex functions $f$ defined on the open interval $(0, \infty)$. Boundedness is not essential on $(0,1]$, but is on $[1, \infty)$. See Lemma 2.3 below.

Likewise Theorem 2.1 can be modified to cover functions $f$ defined on $(0, \infty)$ which are nonincreasing and convex. Such functions can be approximated from below by functions of the type described in Theorem 2.1; and through use of the monotone convergence theorem, we can obtain:

Corollary 2.1. If, in addition to $(2.1), \mathrm{P}(\mathrm{Z}=(0,0))=0$, then $\left(\mathrm{P}\left(\mathrm{Z}^{\prime}=(0,0)\right)=0\right.$ and) (2.2) holds for each nonincreasing convex function $f$ on $(0, \infty)$ for which the expectations contained therein exist. 
It is apparent from the nature of assumption (2.1), appearing in Theorem 2.1 , that inequality (2.2) can be extended to

$$
E f\left(\alpha X^{2}+\beta Y^{2}\right) \geq E f\left(\alpha X^{2}+\beta Y,{ }^{2}\right), \quad \alpha \geq 0, \quad \beta \geq 0 .
$$

There are, of course, many nonincreasing convex functions $f$ to which Theorem 2.1 or its corollary is applicable. As an example the assumptions of Theorem 2.1 imply $E R^{\alpha} \leq E R^{\prime \alpha}, 0<\alpha \leq 2$, where $R^{2}=X^{2}+Y^{2}$ and $R^{\prime 2}=X^{\prime 2}+Y^{\prime 2}$, while the assumptions of Corollary 2.1 permit the conclusion $E R^{\alpha} \geq E R^{\alpha}, \alpha<0$.

The value of Theorem 2.1 and its corollary depends, of course, upon the reasonableness of assumption (2.1), an inequality of type (1.1). Theorem 2.1 of Das Gupta et a1. [3] states easily checked conditions under which this inequality holds for pairs of related elliptically contoured distributions, as we11 as conditions under which assumption (2.7) holds in Theorem 2.2 below and in its corollary.

The requirements in Theorem 2.1 that $f$ be nonincreasing and convex are both necessary for the generality of the theorem: If $f$ is any function on $[0, \infty)$ which satisfies (2.2) whenever (2.1) holds and the expectations make sense, then $f$ must be nonincreasing and convex.

Proof. The need for $f$ to be nonincreasing can be seen by considering nonstochastic $Z$ and $Z^{\prime}$ of the form $(x, 0)$ and $\left(x^{\prime}, 0\right), 0 \leq x \leq x^{\prime}<\infty$. Now suppose $f$ is nonincreasing and satisfies (2.2) for all $Z=(X, Y)$ and $Z^{\prime}=\left(X^{\prime}, Y^{\prime}\right)$ satisfying $(2.1)$. For $s>0$ and $\rho \in(0,1]$, let $Z^{\prime}=s^{\frac{1}{2}} V$ and $Z=s^{\frac{1}{2}} V\left(\begin{array}{ll}1 & \rho \\ \rho & 1\end{array}\right)^{\frac{1}{2}}$, where $V$ is uniformly distributed on the unit circle. Since $Z$ and $Z^{\prime}$ are elliptically contoured vectors which satisfy (2.1) (cf. Theorem 2.1 of [3]), inequality (2.2) holds, which translates into

$$
f(s) \leq \pi^{-1} \int_{-1}^{1}\left(1-u^{2}\right)^{-\frac{1}{2}} f(s[1+u \rho]) d u, \quad s>0, \quad \rho \in(0,1]
$$


Replacing $s$ by $s-1 / n$ and letting $n \rightarrow \infty$ yields

$$
f(s-) \leq \pi^{-1} \int_{-1}^{1}\left(1-u^{2}\right)^{-\frac{1}{2}} f(s[1+u \rho]) d u, \quad s>0, \quad \rho \in(0,1]
$$

which, in turn, due to the monotonicity of $f$, yields

$$
f(s-) \leq \pi^{-1} \int_{-1}^{0}\left(1-u^{2}\right)^{-\frac{1}{2}} f(s-s \rho) d u+\int_{0}^{1}\left(1-u^{2}\right)^{-\frac{1}{2}} f(s+) d u=\frac{1}{2} f(s-s \rho)+\frac{1}{2} f(s+)
$$

for $s>0$ and $\rho \in(0,1]$. Letting $\rho \downarrow 0$, we obtain $f\left(s^{+}\right) \geq f\left(s_{-}\right), s>0$, which establishes the continuity of $f$ on $(0, \infty)$.

Now suppose $f$ is not convex so that for some $0 \leq a<b$, we have $f(a)>f(b)$

and

$$
f(a)+f(b)<2 f\left(\frac{a+b}{2}\right) \text {. }
$$

Consider lines $t=m s+c, a \leq s \leq b$, of negative slope $m=(f(b)-f(a)) /(b-a)$. For large values of $c$, the line $t=m s+c>f(s)$ over the entire interval $[a, b]$. Let $c$ decrease until the line first touches the graph of $f$ at some point in the interval $[a, b]$, and 1 et $s_{0}$ be the smallest such point of contact with this line. (Since $f$ is continuous on $(0, \infty)$, both $c$ and $s_{0}$ are well-defined.) Due to $(2.6), s_{0}$ is in the open interval $(a, b)$. Setting $s=s_{0}$ and $\rho=\left(1-\frac{a}{s_{0}}\right) \wedge\left(\frac{b}{s_{0}}-1\right)$, so that $0 \leq \rho \leq 1$ and $a \leq s(1+u \rho) \leq b$ for $-1 \leq u \leq 1$, we obtain from inequality $(2.5)$ :

$$
\begin{aligned}
f\left(s_{0}\right) & \leq \pi^{-1} \int_{-1}^{1}\left(1-u^{2}\right)^{-\frac{1}{2}} f\left(s_{0}[1+u \rho]\right) d u \leq \pi^{-1} \int_{-1}^{1}\left(1-u^{2}\right)^{-\frac{1}{2}}\left(m s_{0}[1+u \rho]+c\right) d u \\
& =m s_{0}+c=f\left(s_{0}\right) .
\end{aligned}
$$

This can only happen if

$$
f\left(s_{0}[1+u p]\right)=m s_{0}(1+u p)+c, \quad-1 \leq u \leq 1,
$$


which is impossible (for negative $u$ ) due to the way $s_{0}$ is defined. Thus $f$ must be convex.

We remark that the random variables $R^{2}$ and $R^{\prime 2}$, associated with the random vectors $Z=s^{\frac{1}{2}} V\left(\begin{array}{ll}1 & \rho \\ \rho & 1\end{array}\right)^{\frac{1}{2}}$ and $Z^{\prime}=s^{\frac{1}{2}} V$ (used in this proof), are not stochastically ordered since $E R^{2}=E R^{\prime}=s$. Thus condition (2.1) can hold without $\mathrm{R}^{2}$ being stochastically smaller than $\mathrm{R}^{\prime}{ }^{2}$. It follows, of course, that condition (2.1) can hold without (2.2) holding for every nonincreasing function $f$.

Finally it should be pointed out that the argument used in establishing Theorem 2.1 shows that inequality (1.2) holds for all functions $\mathrm{k}$ of the form

$$
k(x, y)=\int_{0}^{\frac{\pi}{2}} F_{\theta}\left(\frac{|x|}{\cos \theta} \vee \frac{|y|}{\sin \theta}\right) d \mu(\theta)
$$

where $F_{\theta}(r)$ is jointly measurable in $(\theta, r)$ and nonincreasing in $r$ for each fixed $\theta, \mu$ is a measure on the open interval $\left(0, \frac{\pi}{2}\right)$, and the indicated integral exists and is finite. By choosing for instance $F_{\theta}(r)=h(r) g(\theta)$ with $h$ bounded and nonincreasing and $g$ bounded and $\geq 0\left(\right.$ e.g. $\left.g(\theta)=(\sin \theta)^{n}(\cos \theta)^{\mathrm{m}}\right)$ and $\mu$ Lebesgue measure, we can generate a large class of symmetric as well as nonsymmetric functions $\mathrm{k}(\mathrm{x}, \mathrm{y})$. The choice $\mathrm{g}(\theta)=\sin \theta \cos \theta$ gives Theorem 2.1 for bounded nonincreasing convex functions of $x^{2}+y^{2} ; k(x, y)=f\left(x^{2}+y^{2}\right)$.

Theorem 2.1 can be generalized to higher dimensional vectors, and this is done in Theorem 2.2 where the following terminology is used. For $2 \leq n<\infty$, a function $f$ defined on $[0, \infty)$ or $(0, \infty)$ is said to be n-monotone if its $k^{\text {th }}$ order divided differences are of alternating signs for $1 \leq k \leq n$, of nompositive sign for odd $k$ and of nonnegative sign for even $k$. (Thus $\left[x_{0}, x_{1} ; f\right]$, defined by $\left(f\left(x_{0}\right)-f\left(x_{1}\right)\right) /\left(x_{0}-x_{1}\right)$, is nonpositive for distinct $x_{0}$ and $x_{1}$ in the domain of $f ;\left[x_{0}, x_{1}, x_{2} ; f\right]$, defined by $\left(\left[x_{0}, x_{1} ; f\right]-\left[x_{1}, x_{2} ; f\right]\right) /\left(x_{0}-x_{2}\right)$, is nonnegative for distinct $x_{0}, x_{1}$ and $x_{2}$; etc.) It follows from Theorem $A$, page 238, of Roberts 
and Varberg [7] that $f$ is n-monotone iff (i) it is nonincreasing on its domain, (ii) it is (n-2)-times continuously differentiable on $(0, \infty)$ with

$$
(-1)^{k} f^{(k)}(s) \geq 0, \quad s>0, \quad k=1, \ldots, n-2,
$$

and (iii) $(-1)^{n-2} f^{(n-2)}$ is nonincreasing and convex on $(0, \infty)$. For future reference, we note that (iii) is equivalent to: (iii') $(-1)^{n-2} f^{(n-2)}$ is locally absolutely continuous with a nonpositive and nondecreasing (Radon-Nikodym) derivative $(-1)^{n-2} f^{(n-1)}$. A function $f$ defined on $[0, \infty)$ or $(0, \infty)$ is said to be $\infty$-monotone if it is n-monotone for all $n$, i.e., if $f$ is nonincreasing on its domain, and $f$ is infinitely differentiable on $(0, \infty)$ with $(-1)^{k} f^{k}(s) \geq 0, s>0, k \geq 1$. In Lemma 2.3 an integral representation is obtained for all bounded n-monotone functions defined on $[0, \infty), 2 \leq n \leq \infty$. A well-known related notion is that of complete monotonicity. A function $f$ defined on $[0, \infty)$ or $(0, \infty)$ is called completely monotone if it is continuous on its domain, and it is infinitely differentiable on $(0, \infty)$ with $(-1) \mathrm{k}^{(\mathrm{k})}(\mathrm{s}) \geq 0, \mathrm{~s}>0, \mathrm{k} \geq 0$. Thus a completely monotone function is $\infty$-monotone, and if $f$ is $\infty$-monotone on $[0, \infty)$ or $(0, \infty)$, then $-f^{(1)}$ is completely monotone on $(0, \infty)$. Completely monotone functions on $[0, \infty)$ are Laplace transforms of finite measures on $[0, \infty)$, and completely monotone functions on $(0, \infty)$ are Laplace transforms of (not necessarily finite) measures on $[0, \infty)$ for which the Laplace transform is finite on $(0, \infty)$. (See Widder [13].)

Theorem 2.2. If the random vectors $Z=\left(z_{1}, \ldots, z_{n}\right)$ and $Z^{\prime}=\left(z_{1}^{\prime}, \ldots, z_{n}^{\prime}\right), n \geq 2$, satisfy

$$
P\left(\left|z_{1}\right| \leq a_{1}, \ldots,\left|z_{n}\right| \leq a_{n}\right) \geq P\left(\left|z_{1}^{\prime}\right| \leq a_{1}, \ldots,\left|z_{n}^{\prime}\right| \leq a_{n}\right), \quad a_{1} \geq 0, \ldots, a_{n} \geq 0
$$


and if $\mathrm{f}$ is an n-monotone function on $[0, \infty)$, then

$$
\operatorname{Ef}\left(\sum_{1}^{n} z_{i}^{2}\right) \geq \operatorname{Ef}\left(\begin{array}{ll}
n & z_{i}^{\prime 2} \\
1 &
\end{array}\right)
$$

Remarks. 1. Theorem 2.2 can be viewed as an extension to higher dimensions of a we11-known result in one dimension, provided one interprets a 1-monotone function as a nonincreasing function.

2. Some examples of $\infty$-monotone functions on $[0, \infty)$, to which Theorem 2.2 is applicable are: $e^{-\alpha s}(\alpha>0),-s^{\alpha}(0<\alpha \leq 1)$, $(s+a)^{\alpha}(\alpha<0, a>0),-\log (s+a)(a>0)$.

3. An example of an n-monotone function which is not $(n+1)$-monotone is the function $f$ defined by $f(s)=((1-s) \vee 0)^{n-1}, s \geq 0(n \geq 2)$.

Before we prove Theorem 2.2, we must gather together a number of facts, some of which we state in the form of lemmas.

Lemma 2.2. If $\mathrm{h}$ is a bounded function and the random vector $\left(\mathrm{V}_{1}, \ldots, \mathrm{V}_{\mathrm{n}}\right), \mathrm{n} \geq 2$, is uniformly distributed on the surface of the n-dimensionat unit sphere, then

$$
\operatorname{Eh}\left(\frac{x_{1}^{2}}{v_{1}^{2}} v \ldots v \frac{x_{n}^{2}}{v_{n}^{2}}\right)\left|v_{1} \ldots v_{n}\right|=\frac{\Gamma\left(\frac{n}{2}\right)}{\pi^{n / 2}(n-2) !} \int_{1}^{\infty} \frac{(u-1)^{n-2}}{u^{n}} h\left(r^{2} u\right) d u
$$

for every real vector $\left(x_{1}, \ldots, x_{n}\right)$, where $r^{2}=x_{1}^{2}+\ldots+x_{n}^{2}$.

Proof. For $n=2$, (2.9) is established in the proof of Theorem 2.1. We now assume (2.9) is true when $n$ in (2.9) is replaced by $n-1$, and proceed to establish its validity for $n(n \geq 3)$. Using the facts that $v_{n}$ has density 


$$
f_{V_{n}}(v)=\frac{\Gamma\left(\frac{n}{2}\right)}{\pi^{\frac{1}{2}} \Gamma\left(\frac{n-1}{2}\right)}\left(1-v^{2}\right)^{\frac{n-3}{2}}, \quad-1<v<1,
$$

that, conditioned on $v_{n}=v,\left(1-v^{2}\right)^{-\frac{1}{2}}\left(v_{1}, \ldots, v_{n-1}\right)$ is uniformly distributed on the (n-1)-dimensional unit sphere (see for instance Lemma 3 in [1]), and therefore that (by the induction hypothesis) the conditional expectation of

$$
\begin{aligned}
& h\left(\frac{x_{1}^{2}}{v_{1}^{2}} v \ldots v \frac{x_{n}^{2}}{v_{n}^{2}}\right)\left|v_{1} \ldots v_{n}\right| \text { given } v_{n}=v \text { is } \\
& \qquad \frac{\Gamma\left(\frac{n-1}{2}\right)}{\frac{n-1}{2}(n-3) !} v\left(1-v^{2}\right)^{\frac{n-1}{2}} \int_{1}^{\infty} \frac{(u-1)^{n-3}}{u^{n-1}} h\left(\frac{\left(r^{2}-x_{n}^{2}\right) u}{1-v^{2}} v \frac{x_{n}^{2}}{v^{2}}\right) d u,
\end{aligned}
$$

we obtain (after some minor simplifications)

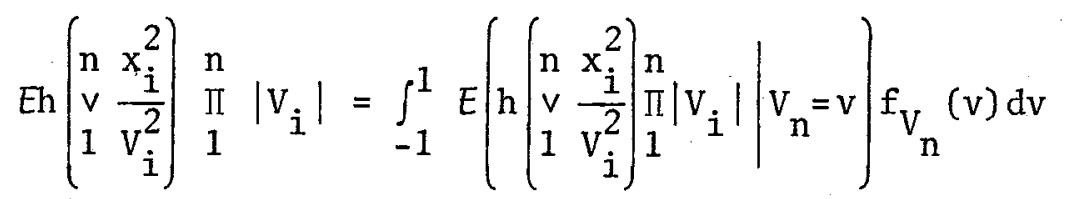

$$
\begin{aligned}
& =\frac{\Gamma\left(\frac{n}{2}\right)}{\pi^{n / 2}(n-3) !} \int_{1}^{\infty} \frac{(u-1)^{n-3}}{u^{n-1}} \int_{0}^{1} h\left(\frac{\left(r^{2}-x_{n}^{2}\right) u}{1-v^{2}} v \frac{x_{n}^{2}}{v^{2}}\right) v\left(1-v^{2}\right)^{n-2} d v d u \text {. }
\end{aligned}
$$

With the change of variable $v \rightarrow y: \frac{u\left(r^{2}-x_{n}^{2}\right)}{1-v^{2}} v \frac{x_{n}^{2}}{v^{2}}=r^{2} y$, the inner integral in (2.10) simplifies to

$$
\frac{u^{n-1}}{r^{2(n-1)}} \frac{u\left(r^{2}-x_{n}^{2}\right)+x_{n}^{2}}{r^{2}} h\left(r^{2} y\right)\left\{\frac{x_{n}^{2}\left(r^{2} y-x_{n}^{2}\right)^{n-2}}{u^{n-1}}+\left(r^{2}-x_{n}^{2}\right)^{n-1}\right\} y^{-n} d y
$$

and (2.10) becomes 


$$
\frac{I^{\prime}\left(\frac{n}{2}\right)}{\frac{n}{2}(n-3) !} \cdot r^{-2(n-1)} \int_{1}^{\infty} \frac{h\left(r^{2} y\right)}{y^{n}} \int_{1}^{\frac{r^{2} y-x_{n}^{2}}{r^{2}-x_{n}^{2}}}(u-1)^{n-3}\left\{\frac{x_{n}^{2}\left(r^{2} y-x_{n}^{2}\right)}{u^{n-1}}+\left(r^{2}-x_{n}^{2}\right)^{n-1}\right\} d u d y .
$$

The inner integral equals

$$
\begin{aligned}
x_{n}\left(r^{2} y-x_{n}^{2}\right) \frac{1}{n-2}\left(1-\frac{r^{2}-x_{n}^{2}}{r^{2} y-x_{n}^{2}}\right)^{n-2} & +\left(r^{2}-x_{n}^{2}\right)^{n-1} \frac{1}{n-2}\left(\frac{r^{2} y-x_{n}^{2}}{r^{2}-x_{n}^{2}-1}\right)^{n-2} \\
& =\frac{1}{n-2} r^{2(n-1)}(y-1)^{n-2}
\end{aligned}
$$

and thus (2.10) becomes

$$
\frac{\Gamma\left(\frac{n}{2}\right)}{\pi^{n / 2}(n-2) !} \int_{1}^{\infty} \frac{(y-1)^{n-2}}{y^{n}} h\left(r^{2} y\right) d y .
$$

By using the same argument used in proving Theorem 2.1, together with Lemma 2.2, one can readily establish (2.8) for functions $f$ defined on $[0, \infty)$ of the form

$$
f(s)=\int_{1}^{\infty} \frac{(u-1)^{n-2}}{u^{n}} h(u s) d u, \quad s \geq 0,
$$

where $h$ is bounded and nonincreasing. The class of such functions is characterized in Lemma 2.3, which follows.

Lemma 2.3. The class $F_{n}$ of functions $f$ described by (2.11), with $\mathrm{h}$ any bounded and nonincreasing function on $[0, \infty)$, coincides with the class of bounded n-monotone functions on $[0, \infty)$. The class $F_{n}^{\prime}$ of functions $f$ of the form 


$$
f(s)=s \int_{s}^{\infty} \frac{(v-s)^{n-2}}{v^{n}} h(v) d v, \quad s>0,
$$

with $h$ any noninereasing function on $(0, \infty)$ and bounded on $[1, \infty)$, coincides with the class of n-monotone functions on $(0, \infty)$ which are bounded on $[1, \infty)(\mathrm{n} \geq 2)$.

Remark. An immediate consequence of this lemma is that the classes $F_{n}$ and $F_{n}$ are nonincreasing in $n$. If $f \in F_{n}\left(\in F_{n}^{\prime}\right)$ for every $n$, then $f-f(\infty)$ is a completely monotone function on $[0, \infty)$ (on $(0, \infty)$ ), and, therefore, $f$ must be of the form

$$
f(s)=c+\int_{0}^{\infty} e^{-s u} d \mu(u), \quad s \geq 0(s>0),
$$

where $c$ is any real number and $\mu$ is a finite measure on $[0, \infty)(\mu$ is a measure on $[0, \infty)$ for which the integral is finite for all $s>0$ ).

Proof. We shall prove the characterization of $F_{n}^{\prime}$. Since the right-hand side of (2.12) is equal to the integral in (2.11) when $s>0$, the stated characterization of $F_{n}$ is easily inferred from that for $F_{n}^{\prime}$.

Suppose $f \in F_{n}^{\prime}$. It is clear from (2.11) that $f$ is bounded on $[1, \infty)$, and from (2.12) that $f$ is $(n-2)$-times continuously differentiable on $(0, \infty)$ with

$$
\begin{array}{r}
f^{(k)}(s)=(-1)^{k-1} \frac{(n-2) !}{(n-k) !} \int_{s}^{\infty} \frac{(v-s)^{n-k-2}}{v^{n}}[k v-(n-1) s] h(v) d v, \\
s>0, \quad 1 \leq k \leq n-2 .
\end{array}
$$

Also $f^{(n-2)}$ is locally absolutely continuous with a (Radon-Nikodym) derivative

$$
f^{(n-1)}(s)=(-1)^{n-2}(n-1) ! \int_{s}^{\infty} \frac{h(v)}{v^{n}} d v+(-1)^{n-1}(n-2) ! s^{-(n-1)} h(s) \text { a.e. on }(0, \infty,
$$


which, after integrating by parts, simplifies to the version we will use:

$$
f^{(n-1)}(s)=(-1)^{n}(n-2) ! \int_{s}^{\infty} v^{-(n-1)} d h(v), \quad s>0
$$

Clearly $f^{(n-1)}(\infty)\left(=\lim _{s \rightarrow \infty} f^{(n-1)}(s)\right)$ exists and equals zero. In fact,

$$
\mathrm{s}^{\mathrm{k}-1} \mathrm{f}^{(\mathrm{k})}(\mathrm{s}) \rightarrow 0 \text { as } \mathrm{s} \rightarrow \infty, \quad 1 \leq \mathrm{k} \leq \mathrm{n}-1
$$

This is obvious from (2.14) for $k=n-1$ and from (2.13) for $1 \leq k \leq n-2$. Observe that $(-1)^{n-1} f^{(n-1)}$ is nonincreasing. Since $h$ is nonincreasing, it follows by $(2.14)$ that $(-1)^{n-1} f^{(n-1)}$ is nonnegative. Proceeding by backwards induction: From the nonnegativity of $(-1)^{n-1} f^{(n-1)}$, we infer that $(-1)^{n-2} f^{(n-2)}$ is nonincreasing. Since $\mathrm{f}^{(\mathrm{n}-2)}(\infty)=0$ (implied by $\left.(2.15)\right),(-1)^{n-2} f^{(n-2)}$ is nonnegative, etc. Thus $(-1)^{j_{f}(j)}$ is nonnegative for $j=1, \ldots, n-1$, and $(-1)^{n-1} f^{(n-1)}$ is nonincreasing, which together say that $f$ is n-monotone. Conversely, suppose $f$ is n-monotone on $(0, \infty)$ and bounded on $[1, \infty)$. Define $\mathrm{h}$ on $(0, \infty)$ by

$$
h(v)=(n-1) \sum_{k=0}^{n-1} \frac{(-1)^{k}}{k !} v^{k}{ }^{(k)}(v), \quad v>0
$$

which is nonincreasing because each term in the sum is nonincreasing (a consequence of $f$ being n-monotone). Observe that $h$ is of bounded local variations and

$$
d h(v)=\frac{(-1)^{n-1}}{(n-2) !} v^{n-1} d f^{(n-1)}(v)
$$

i.e., 


$$
h(v)-h(s)=\frac{(-1)^{n-1}}{(n-2) !} \int_{s}^{v} u^{n-1} d f^{(n-1)}(u), \quad 0<s \leq v<\infty
$$

Then for $s>0$ (using $(2.18)$ ),

$$
\begin{aligned}
s \int_{s}^{\infty} \frac{(v-s)^{n-2}}{v^{n}}(h(v)-h(s)) d v & =\frac{(-1)^{n-1}}{(n-2) !} s \int_{s}^{\infty} \frac{(v-s)^{n-2}}{v^{n}} \int_{s}^{v} u^{n-1} d f^{(n-1)}(u) d v \\
& =\frac{(-1)^{n-1}}{(n-1) !} \int_{s}^{\infty}\left\{u^{n-1}-(u-s)^{n-1}\right\} d f^{(n-1)}(u) \equiv F_{n-1}(s),
\end{aligned}
$$

say, where we have applied Fubini's theorem for nonnegative functions (without knowing, as yet, that $\mathrm{F}_{\mathrm{n}-1}(\mathrm{~s})$ is finite). In what follows, we shall need to use (2.15), which should be justified in the present context. This is done in Lemma 2.4 below for the $\mathrm{k}^{\text {th }}$ derivative of an arbitrary $\mathrm{n}$-monotone function, $2 \leq k \leq n-1$. The remainder of (2.15), for $k=1$, is valid in the present context since, by assumption, $f$ is bounded on $[1, \infty)$.

Now, using integration by parts and (2.15) for $k=n-1$, we obtain

$$
F_{n-1}(s)=-\frac{(-1)^{n-1}}{(n-1) !} s^{n-1} f^{(n-1)}(s)+F_{n-2}(s),
$$

where

$$
F_{n-2}(s)=\frac{(-1)^{n-2}}{(n-2) !} \int_{s}^{\infty}\left\{u^{n-2}-(u-s)^{n-2}\right\} f^{(n-1)}(u) d u
$$

Proceeding by backwards induction, we are eventually led to

$$
s \int_{s}^{\infty} \frac{(v-s)^{n-2}}{v^{n}}(h(v)-h(s)) d v=f(s)-\sum_{k=0}^{n-1} \frac{(-1)^{k}}{k !} s^{k} f^{(k)}(s),
$$


which, in view of (2.16), establishes (2.12). The boundedness of $h$ on $[1, \infty)$ may be inferred from (2.12).

If $f$ is $n$-monotone on $[0, \infty)$ or $(0, \infty)$, then $(-1)^{k_{f}}(k)$ is nonnegative and nonincreasing on $(0, \infty)$ for $k=1, \ldots, n-1$, and, hence,

$$
\begin{aligned}
0 & \leq s(-1)^{k}{ }^{(k)}(s) \leq \int_{s / 2}^{s}(-1)^{k} f^{(k)}(u) d u \\
& \leq 2\left\{(-1)^{k} f^{(k-1)}(s)-(-1)^{k} f^{(k-1)}(s / 2)\right\}, \quad s>0, \quad 1 \leq k \leq n-1 .
\end{aligned}
$$

These inequalities permit us to describe the behavior of the derivatives of $f$ as $\mathbf{s} \rightarrow \infty$ and $\mathbf{s}+0$ :

Lemma 2.4. If $\mathrm{f}$ is $\mathrm{n}$-monotone on $[0, \infty)$ or $(0, \infty)$ for some $\mathrm{n} \geq 3$, then

$$
\mathrm{s}^{\mathrm{k}-1} \mathrm{f}^{(\mathrm{k})}(\mathrm{s}) \rightarrow 0 \text { as } \mathrm{s} \rightarrow \infty, \quad 2 \leq k \leq \mathrm{n}-1 \text {. }
$$

Proof. This follows by induction from (2.19), provided (corresponding to $\mathrm{k}-1=1)$

$$
f^{(1)}(s)-f^{(1)}(s / 2) \rightarrow 0
$$

But this is the case since $f^{(1)}$ is nonpositive and nondecreasing.

Lemma 2.5. If $f$ is n-monotone on $[0, \infty)$, or n-monotone on $(0, \infty)$ with $f(0+)$ finite, for some $\mathrm{n} \geq 2$, then

$$
\mathrm{s}^{\mathrm{k}} \mathrm{f}^{(\mathrm{k})}(\mathrm{s}) \rightarrow 0 \text { as } \mathrm{s} \downarrow 0, \quad \mathrm{I} \leq \mathrm{k} \leq \mathrm{n}-1
$$

Proof. In either case, $f\left(0_{+}\right)$exists and is finite. Thus $f(s)-f(s / 2) \rightarrow 0$ as $\mathrm{s} \downarrow 0$, and (2.21) follows from $(2.19)$ by induction. 
Proof of Theorem 2.2. In view of the remark preceding Lemma 2.3, we may take (2.8) to be established for all bounded n-monotone functions $f$ on $[0, \infty)$. The proof for unbounded $f$ requires the removal from $f$ of its (possible) linear part and then a truncation argument.

Suppose first that $f(s)=-c s, s \geq 0$, where $c>0$. Inequality (2.8) can be expressed as

$$
\sum_{i}^{n} E Z_{i}^{2} \leq \sum_{1}^{n} E Z_{i}^{\prime 2}
$$

which must hold since (2.7) implies $z_{i}^{2}$ is stochastically smaller than $z_{i}^{2}$, $\mathrm{i}=1, \ldots, \mathrm{n}$.

Now suppose $f$ is any unbounded n-monotone function on $[0, \infty)$, i.e. $f(\infty)=-\infty$. Since $f^{(1)}$ is nonpositive and nondecreasing, the finite nonpositive limit $f^{(1)}(\infty)$ exists. We sha11 assume, without loss of generality, that $f^{(1)}(\infty)=0$. For otherwise, we may express $f$ as the sum of two n-monotone functions, $f=f_{1}+f_{2}$ where $f_{1}^{(1)}(\infty)=0$ and $f_{2}(s)=f^{(1)}(\infty) \cdot s, s \geq 0$, and treat the parts independently. We shall truncate $f$ as follows: Define $h$ on $(0, \infty)$ by (2.16) and $h(0)=(n-1) f(0)$. For $x>0$, let $h_{x}(v)=h(v \wedge x), v \geq 0$, and define $f_{x}$ by $(\sec (2.11))$

$$
f_{x}(s)=\int_{1}^{\infty} \frac{(u-1)^{n-2}}{u^{n}} h_{x}(u s) d u, \quad s \geq 0 .
$$

Since $h$ is nonincreasing on $(0, \infty)$ (see $(2.17)$ ) and $h(0+)=(n-1) f(0+) \leq(n-1) f(0)=h(0)$ (cf. Lemma 2.5), it follows that $h$ is nonincreasing on $[0, \infty)$ and, consequently, $h_{x}$ is a bounded nonincreasing function on $[0, \infty)$ for every $x>0$. This implies that (2.8) holds for each 
function $f_{x}$, and it only remains to show, if possible, that $f_{x} \downarrow f$ as $x \rightarrow \infty$ (so that (2.8) follows for $f$ itself by the monotone convergence theorem). Since $h_{x}$ is nonincreasing in $x$, so is $f_{x}$ (apparent from $(2.22)$ ), and thus it is only necessary to show the pointwise convergence of $f_{x}$ to $f$.

From (2.22) we have $f_{x}(0)=(n-1) h(0)=f(0)$ for all $x>0$. Thus we may focus our attention exclusively on points $s>0$. For such points, it is more convenient to use the following variant of (2.22) (see (2.12)):

$$
f_{x}(s)=s \int_{s}^{\infty} \frac{(v-s)^{n-2}}{v^{n}} h_{x}(v) d v, \quad s>0
$$

For $\mathrm{x}>\mathrm{s}>0$, we have (using (2.18))

$$
\begin{aligned}
f_{x}(s) & =s \int_{s}^{x} \frac{(v-s)^{n-2}}{v^{n}} h(v) d v+s \int_{s}^{\infty} \frac{(v-s)^{n-2}}{v^{n}} d v \cdot h(x) \\
& =s \int_{s}^{x} \frac{(v-s)^{n-2}}{v^{n}}\left\{\frac{(-1)^{n-1}}{(n-2) !} \int_{s}^{v} u^{n-1} d f^{(n-1)}(u)\right\} d v \\
& +\frac{h(x)}{(n-1)}\left\{1-\left(1-\frac{s}{x}\right)^{n-1}\right\}+\frac{h(s)}{n-1}\left(1-\frac{s}{x}\right)^{n-1} \\
& =\frac{(-1)^{n-1}}{(n-1) !} \int_{s}^{x} u^{n-1}\left\{\left(1-\frac{s}{x}\right)^{n-1}-\left\{1-\frac{s}{u}\right)^{n-1}\right\} d f(n-1)(u) \\
& =-\frac{(-1)^{n-1}}{(n-1) !} \int_{s}^{x}(u-s)^{n-1} d f^{(n-1)}(u)+\frac{h(x)}{n-1} \cdot
\end{aligned}
$$

By repeatedly integrating by parts (much as in the proof of Lemma 2.3), we obtain 


$$
f_{x}(s)=f(s)+\sum_{k=1}^{n-1} \frac{(-1)^{k}}{k !} f^{(k)}(x)\left\{x^{k}-(x-s)^{k}\right\}, \quad x>s>0
$$

Since, as $x \rightarrow \infty, f^{(1)}(x) \rightarrow f^{(1)}(\infty)=0$ (assumed without loss of generality), it follows from Lemma 2.4 that the sum converges to zero as $x \rightarrow \infty$. Thus $f_{x}(s) \rightarrow f(s)$ as $x \rightarrow \infty$, which completes the proof.

Theorem 2.2 can be extended to n-monotone functions on $(0, \infty)$, to allow for functions which are unbounded at zero as well as at infinity.

Corollary 2.2. If, in addition to (2.7), $\mathrm{P}\left(\mathrm{z}_{1}=0, \ldots, \mathrm{z}_{\mathrm{n}}=0\right)=0$, then $\left(P\left(Z_{1}^{\prime}=0, \ldots, Z_{n}^{\prime}=0\right)=0\right.$ and $)(2.8)$ holds for each $n$-monotone function $f$ on $(0, \infty)$ for which the expectations in (2.8) are defined.

Proof. Let $f$ be n-monotone on $(0, \infty)$ with $f(0+)=\infty$ and $f(\infty)=-\infty$. (Functions $f$ with smaller ranges can be handled similarly or more easily.) Let $s_{0}$ be the zero of $f, f\left(s_{0}\right)=0$, and for each $k>\left(2 / s_{0}\right)$ define $f_{k}(s)=f\left(s+\frac{1}{k}\right), s \geq 0$. Then each $f_{k}$ is n-monotone on $[0, \infty)$, and by Theorem 2.2, $E f_{k}\left(|| z||^{2}\right) \geq E f_{k}\left(|| z^{\prime}||^{2}\right)$. Also as $k \uparrow \infty, f_{k} \uparrow f$ on $(0, \infty)$. More precisely, $f_{k}^{+} \uparrow f^{+}$and by monotone convergence $E f_{k}^{+}\left(\|z\|^{2}\right) \uparrow E f^{+}\left(\|z\|^{2}\right)$. Also for $s \geq s_{0}-\frac{1}{k}$, since $0 \leq-f^{(1)} \downarrow$, we have

$$
\begin{aligned}
0 \leq f_{k}^{-}(s)-f^{-}(s) & \leq-f\left(s+\frac{1}{k}\right)+f(s)=-\int_{s}^{s+\frac{1}{k}} f^{(1)}(u) d u \\
& \leq-f^{(1)}\left(s_{0}-\frac{1}{k}\right) \frac{1}{k} \leq \frac{1}{k}\left|f^{(1)}\left(\frac{s_{0}}{2}\right)\right|
\end{aligned}
$$

and thus $0 \leq f_{k}^{-}(s)-f^{-}(s) \leq \frac{1}{k}\left|f^{(1)}\left(\frac{s}{2}\right)\right|, s \geq 0$. It follows that $E f_{k}^{-}\left(|| z||^{2}\right)$ and $E f^{-}\left(|| z||^{2}\right)$ are finite or infinite together and thus $E f_{k}^{-}\left(\|z\|^{2}\right) \downarrow E f^{-}\left(\| z||^{2}\right)$ 
(by dominated convergence if they are finite or trivially if they are infinite). Since $E f\left(|| Z||^{2}\right)$ is defined by $E f^{+}\left(\left.|| Z\right|^{2}\right)-E f^{-}\left(\|Z\|^{2}\right)$ iff at least one of the two terms if finite, $(2.8)$ follows.

Some examples of an $\infty$-monotone function, to which Corollary 2.2 is applicable, are: $s^{\alpha}(\alpha<0),-\log s$.

We have already shown that the 2-monotone functions provide the appropriate class for the result of Theorem 2.1. The following example shows that 3-monotone functions provide the appropriate class for the result of Theorem 2.2 for $n=3$ (by constructing, for a 2-monotone function which is not 3-monotone, 3-dimensional random vectors $Z$ and $Z^{\prime}$ which satisfy (2.7) and for which (2.8) fails), and we anticipate that similar examples would show the same for $n>3$.

Example. Suppose $f$ is 2 -monotone but not 3 -monotone on $[0, \infty)$. Then for some $a$ and $b, a \geq 0, b>0$, one has

$$
f(a+3 b)-3 f(a+2 b)+3 f(a+b)-f(a)>0 .
$$

(Implicitly we are saying that functions $f$ which are 2 -monotone and satisfy the converse of (2.23) for all $\mathrm{a}$ and $\mathrm{b}$ are 3-monotone, which can be verified.) Let $3 \alpha^{2}=a$ and $2 \alpha^{2}+\beta^{2}=a+b$ be used to define $\alpha$ and $\beta(0 \leq \alpha<\beta)$, and let $z$ and $Z^{\prime}$ be three-dimensional random vectors whose distributions are described by the following table: 


\begin{tabular}{|ccccc|}
\hline$z$ & $P(Z=z)$ & $P\left(Z^{\prime}=z\right)$ & $P(Z \leq z)$ & $P\left(Z^{\prime} \leq z\right)$ \\
\hline$(\alpha, \alpha, \alpha)$ & $\frac{11}{81}$ & $\frac{9}{81}$ & $\frac{11}{81}$ & $\frac{9}{81}$ \\
$(\alpha, \alpha, \beta)$ & $\frac{4}{81}$ & $\frac{6}{81}$ & $\frac{15}{81}$ & $\frac{15}{81}$ \\
$(\alpha, \beta, \alpha)$ & $\frac{4}{81}$ & $\frac{6}{81}$ & $\frac{15}{81}$ & $\frac{15}{81}$ \\
$(\beta, \alpha, \alpha)$ & $\frac{4}{81}$ & $\frac{6}{81}$ & $\frac{15}{81}$ & $\frac{15}{81}$ \\
$(\alpha, \beta, \beta)$ & $\frac{8}{81}$ & $\frac{6}{81}$ & $\frac{27}{81}$ & $\frac{27}{81}$ \\
$(\beta, \alpha, \beta)$ & $\frac{8}{81}$ & $\frac{6}{81}$ & $\frac{27}{81}$ & $\frac{27}{81}$ \\
$(\beta, \beta, \alpha)$ & $\frac{8}{81}$ & $\frac{6}{81}$ & $\frac{27}{81}$ & $\frac{27}{81}$ \\
$(\beta, \beta, \beta)$ & $\frac{34}{81}$ & $\frac{36}{81}$ & 1 & 1 \\
\hline
\end{tabular}

From the last two columns it is apparent that condition (2.7) holds. Now for $\mathrm{R}^{2}=\mathrm{ZZ}^{\mathrm{t}}$ and $\mathrm{R}^{\prime 2}=\mathrm{Z}^{\prime} \mathrm{Z}^{\prime}{ }^{t}$, we have

$$
\begin{aligned}
& E f\left(R^{2}\right)=\frac{11}{81} f(a)+\frac{12}{81} f(a+b)+\frac{24}{81} f(a+2 b)+\frac{34}{81} f(a+3 b), \\
& E f\left(R^{\prime 2}\right)=\frac{9}{81} f(a)+\frac{18}{81} f(a+b)+\frac{18}{81} f(a+2 b)+\frac{36}{81} f(a+3 b)
\end{aligned}
$$

From (2.23) it follows that $E f\left(R^{\prime 2}\right)>E f\left(R^{2}\right)$. Consequently, the assumption of 3-monotonicity in Theorem 2.2 when $Z$ and $Z^{\prime}$ are three-dimensional is essential; it is impossible to consider a larger class of functions.

\section{Expectation inequalities for pairs of functions}

In this section we consider random vectors $Z$ and $Z^{\prime}\left(\right.$ i.e. $R=\mathbb{R}^{\mathrm{n}}$ ) which satisfy (1.1) with $A$ the class of all principal lower and upper ideals $(-\infty, z]$ 
and $[z, \infty), z \in \mathbb{R}^{n}$. When $n=1,(1.1)$ says that $z$ and $z$ ' have the same distribution, which is not interesting. When $n=2,(1.1)$ says that $Z$ and $Z^{\prime}$ have the same marginal distributions and that their bivariate distribution functions $H$ and $H^{\prime}$ satisfy $H^{\prime} \geq H^{\prime}$. Our attention will be focused on the bivariate case, and only at the end of the section will we consider a higher dimensional case.

It is shown in $[2,12]$ that $(1.1) \Longleftrightarrow(1.2)$ with $F_{1}$ the class of all quasi-monotone functions (cf. (1.14)) for which the expectations in (1.2) are defined and which satisfy certain minor regularity conditions. (See

Theorem 1 in [2].) The separation approach yields (1.5.iii) with $F_{2}$ defined by (1.4) as the class of all pairs of functions $l, k$ which can be separated by a quasi-monotone function $m: l=m+f, k=m-g$ where $f$ and $g$ are nonnegative. (Large classes of quasi-monotone functions are known or can be constructed; see for instance [2].) When $\ell$ and $\mathrm{k}$ are separated by a quasi-monotone function then they satisfy (1.15) (cf. (1.4) and (1.14)). However (1.15) is not sufficient for $l$ and $k$ to be separated by a quasi-monotone function: there exist functions $\ell$ and $k$ satisfying (1.15) which are sufficiently close that no quasi-monotone function can exist between them. This is easily demonstrated with the aid of Figure I.

In Figure $I$, relevant values of $k$ and $\ell$ are indicated at various points within an array of eight points possessing a particular geometric orientation in the plane. In order to obtain a contradiction, it is assumed that a quasi-monotone function $m$ satisfying $\ell \geq m \geq k$ does exist. Figure $I$ indicates two points in the array where it is impossible to define $m$ simultaneously. In order to insure that (1.15) is satisfied, it is sufficient to define $k$ as -10 , say, and $\ell$ as 10 at all points in the plane for which an explicit definition is not given in Figure $I$. 


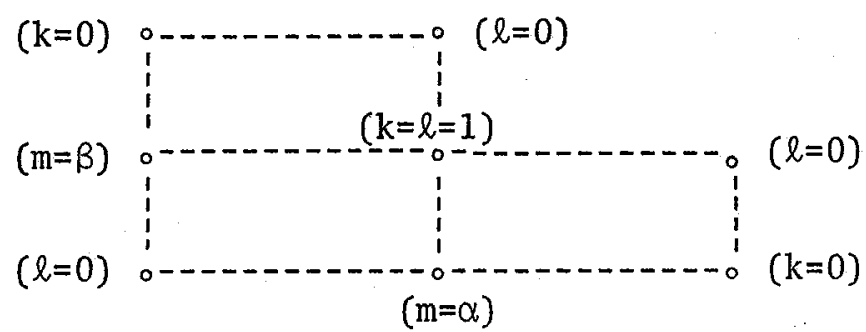

FIGURE I

By referring to the four points in the lower left-hand corner of Figure $I$, one can easily deduce from $l \geq m \geq k$ and the quasi-monotonicity of $m$ that the inequality $\alpha+\beta \leq 1$ must hold. In the same way, one can obtain the contradicting inequalities $\alpha \geq 1$ and $\beta \geq 1$ by examining the four points in the lower right- and upper left-hand corners of Figure I, respectively. Thus no quasi-monotone function $\mathrm{m}$ exists which satisfies $\ell \geq \mathrm{m} \geq \mathrm{k}$. This example suggests the possibility that (1.1) can hold without (1.3) holding for all functions $k$ and $\ell$ which satisfy (1.15) (and for which the expectations in (1.3) are defined). In fact, an example based upon Figure $I$ is easily constructed: Let the distribution of $Z$ assign mass $1 / 3$ to each of the points in Figure $I$ at which $l=0$, and let the distribution of $Z^{\prime}$ assign mass $1 / 3$ to each of the three points in Figure $I$ at which $\mathrm{k}$ is explicitly defined. It is easily checked that (1.1) holds but $E l(Z)<E k\left(Z^{\prime}\right)$.

Thus if $Z$ and $Z$ ' are bivariate random variables with equal marginal distributions and with bivariate distribution functions satisfying $H \geq H^{\prime}$ (i.e. if (1.1) holds), then in general this does not imply that (1.3) holds for all functions $\mathrm{k}$ and $\ell$ satisfying (1.15). We now show that this implication is true in certain special cases, using the variation of the surrogate approach involving condition ( $\mathrm{C} 0$ ), which is described in the introduction. We begin with the normal case. 
Theorem 3.1. Suppose $Z$ and $Z$ ' are bivariate normal random variables with common means and variances and with correlation coefficients $\rho$ and $\rho^{\prime}$ satisfying the inequality $\rho \geq \rho^{\prime}$. Then (1.3) holds for every pair of functions $\mathrm{k}$ and $\mathrm{l}$ which satisfy (1.15) and for which the expectations appearing therein make sense.

Proof. Let $(\mathrm{S}, \mathrm{T}, \mathrm{U})$ be normally distributed with a zero mean vector and the covariance matrix

$$
\Sigma_{(S, T, U)}=\left(\begin{array}{ccc}
1 & \rho & \left(\rho^{\prime}-\rho\right) \\
\rho & 1 & \left(\rho^{\prime}-\rho\right) \\
\left(\rho^{\prime}-\rho\right) & \left(\rho^{\prime}-\rho\right) & 2\left(\rho-\rho^{\prime}\right)
\end{array}\right) .
$$

Define

$$
X_{1}=\mu_{x}+\sigma_{X} S, Y_{1}=\mu_{y}+\sigma_{y} T, X_{2}=\mu_{x}+\sigma_{x}(S+U), Y_{2}=\mu_{y}+\sigma_{y}(T+U)
$$

where $\left(\mu_{x}, \mu_{y}\right)$ is the common mean vector, and $\sigma_{x}^{2}$ and $\sigma_{y}^{2}$ are the common variances of $H$ and $H^{\prime}$. It is easily checked that $\left(X_{1}, Y_{1}\right)$ and $\left(X_{2}, Y_{2}\right)$ have distribution function $H$ and that $\left(X_{1}, Y_{2}\right)$ and $\left(X_{2}, Y_{1}\right)$ have distribution function $H^{\prime}$. Moreover $\left(\mathrm{X}_{2}-\mathrm{X}_{1}\right)\left(\mathrm{Y}_{2}-\mathrm{Y}_{1}\right)=\sigma_{\mathrm{X}} \sigma_{\mathrm{y}} \mathrm{U}^{2} \geq 0$. Thus condition (CO) is satisfied and (1.3) follows from $(1.15)$, via (1.16) as discussed in the introduction.

Thus when $Z$ and $Z$ ' are as in Theorem 3.1 , and $F_{2}$ is the class of all pairs of functions $\ell$ and $k$ satisfying (1.15) and for which the expectations in (1.3) are defined, we have $\rho \geq \rho^{\prime} \Rightarrow(1.3)$. On the other hand we clearly have $(1.3) \Rightarrow(1.2)$ and, as was already mentioned, (1.2) $\Leftrightarrow(1.1)$ which in this case is equivalent to $H^{\prime} \geq H^{\prime}$. Thus $\rho \geq \rho^{\prime} \Rightarrow H^{\prime} \geq H^{\prime}$, which is a special case of an $\mathrm{n}$-dimensional result due to S1epian [9], and which implies $\rho \geq \rho^{\prime} \Leftrightarrow \mathrm{H}^{\prime} \geq \mathrm{H}^{\prime}$. 
It then follows that when $Z$ and $Z^{\prime}$ are bivariate normal variables with common means and variances then

$$
\rho \geq \rho^{\prime} \Leftrightarrow H \geq H^{\prime} \Leftrightarrow(1.2) \Leftrightarrow(1.3)
$$

Theorem 3.1 can be extended to higher dimensions and from normal to elliptically contoured distributions. If $Z$ is an $n$-dimensional random (row) vector and, for some $n$ (row) vector $\mu$ and some $n \times n$ nonnegative definite matrix $\Sigma$, the characteristic function $\phi_{Z-\mu}(s)$ of $z-\mu$ is a function of the quadratic form $s \Sigma s^{t}, \phi_{Z-\mu}(s)=\phi\left(s \Sigma s^{t}\right)$, we say that $Z$ has an elliptically contoured distribution with parameters $\mu, \Sigma$ and $\phi$, and we write $Z \sim \operatorname{EC}_{\mathrm{n}}(\mu, \Sigma, \phi)$. When $\phi(u)=\exp (-\mathrm{u} / 2), \mathrm{EC}_{\mathrm{n}}(\mu, \Sigma, \phi)$ is the normal distribution $\mathrm{N}_{\mathrm{n}}(\mu, \Sigma)$. The location and scale parameters $\mu$ and $\Sigma$ can be any $n$ vector and any $n \times n$ nonnegative definite matrix, while the class $\Phi_{k}$ of admissible functions $\phi$ depends on the rank $k$ of $\Sigma, r(\Sigma)=k$, and consists of all functions of the form

$$
\phi(u)=\int_{[0, \infty)} \Omega_{k}\left(r^{2} u\right) d F(r), \quad u \geq 0
$$

for some distribution function $F$ on $[0, \infty)$, where $\Omega_{k}\left(\|s\|^{2}\right), s \in \mathbb{R}^{k}$, is the characteristic function of the uniform distribution on the surface of the unit sphere of $\mathbb{R}^{k}$. This follows from a theorem of Schoenberg [8] and is discussed in [1] where the following useful stochastic representation is also introduced. Let $\Sigma=A^{t} A$ be a rank factorization of $\Sigma$, i.e. $A$ is $k \times n$ and $r(\Sigma)=k=r(A)$. Then $Z$ has the "canonical" stochastic representation

$$
\mathrm{z} \stackrel{\mathrm{d}}{=} \mu+\mathrm{RU}^{(\mathrm{k})} \mathrm{A}
$$

where the equality is in distribution, $\mathrm{R}$ is a nonnegative random variable (with distribution $F), U^{(k)}$ is a k-dimensional random vector uniformly distributed on 
the surface of the unit sphere in $\mathbb{R}^{k}$, and $R$ and $U^{(k)}$ are independent.

Theorem 3.2. Suppose that $\mathrm{Z} \sim \mathrm{EC}_{2}(\mu, \Sigma, \phi)$ and $\mathrm{Z}^{\prime} \sim \mathrm{EC}_{2}\left(\mu, \Sigma^{\prime}, \phi\right)$ where

$$
\Sigma=\left(\begin{array}{cc}
\sigma_{1}^{2} & \rho \sigma_{1} \sigma_{2} \\
\rho \sigma_{1} \sigma_{2} & \sigma_{2}^{2}
\end{array}\right), \quad \Sigma^{\prime}=\left(\begin{array}{cc}
\sigma_{1}^{2} & \rho^{\prime} \sigma_{1} \sigma_{2} \\
\rho^{\prime} \sigma_{1} \sigma_{2} & \sigma_{2}^{2}
\end{array}\right)
$$

and $\rho \geq \rho^{\prime}$. Then (1.3) holds for every pair of functions $k$ and $\ell$ which satisfy (1.15) and for which the expectations appearing in (1.3) are defined.

Proof. If $\rho=1$ and $\rho^{\prime}=-1$ we have

$$
z^{\mathrm{d}}=\mu+R U^{(1)} \mathrm{A}_{1}, \mathrm{Z}^{\prime} \stackrel{\mathrm{d}}{=} \mu+\mathrm{RU}{ }^{(1)} \mathrm{A}_{1}^{\prime}
$$

where $A_{1}=\left(\sigma_{1}, \sigma_{2}\right), A_{1}^{\prime}=\left(\sigma_{1},-\sigma_{2}\right)$. Since $R$ and $U^{(1)}$ are independent, in order to show $E l(Z) \geq E k\left(Z^{\prime}\right)$ it suffices to show

$$
E \ell\left(\mu+r U^{(1)} A_{1}\right) \geq E k\left(\mu+r U^{(1)} A_{1}^{\prime}\right), \quad r \geq 0
$$

Since $k(\cdot)$ and $\ell(\cdot)$ satisfy $(1.15)$, so do $k(\mu+r \cdot)$ and $\ell(\mu+r \cdot)$ for every $\mu$ and $r \geq 0$. Thus it suffices to show $(3.1)$ when $\mu=0$ and $r=1$, i.e. it suffices to show El( $\left(\mathrm{U}^{(1)} \mathrm{A}_{1}\right) \geq \operatorname{Ek}\left(\mathrm{U}^{(1)} \mathrm{A}_{1}^{\prime}\right)$, which is written as

$$
\frac{1}{2} \ell\left(-\sigma_{1},-\sigma_{2}\right)+\frac{1}{2} \ell\left(\sigma_{1}, \sigma_{2}\right) \geq \frac{1}{2} k\left(-\sigma_{1}, \sigma_{2}\right)+\frac{1}{2} k\left(\sigma_{1},-\sigma_{2}\right)
$$

and follows from $(1.15)$.

Now assume that at least one of $\rho, \rho^{\prime}$ differs from 1 in absolute value. Putting 


$$
A=\left(\begin{array}{ccc}
\sigma_{1} \cos \alpha & \sigma_{2} \sin \alpha \\
\sigma_{1} \sin \alpha & \sigma_{2} \cos \alpha
\end{array}\right) \text { and } A^{\prime}=\left(\begin{array}{ccc}
\sigma_{1} \cos \alpha^{\prime} & \sigma_{2} \sin \alpha^{\prime} \\
\sigma_{1} \sin \alpha^{\prime} & \sigma_{2} \cos \alpha^{\prime}
\end{array}\right) \text {, }
$$

where $\alpha$ and $\alpha^{\prime}, \frac{-\pi}{4} \leq \alpha^{\prime} \leq \alpha \leq \frac{\pi}{4}$, are defined by $\rho=\sin 2 \alpha$ and $\rho^{\prime}=\sin 2 \alpha^{\prime}$, we have $\Sigma=A^{t} A$ and $\Sigma^{\prime}=A^{\prime} A^{\prime}$. When $-1<\rho^{\prime}<\rho<1$ then both $\Sigma, \Sigma^{\prime}$ are full rank and $r(A)=2=r\left(A^{\prime}\right)$ so that $\Sigma=A^{t} A, \Sigma^{\prime}=A^{\prime} t^{\prime}$ ' are rank factorizations of $\Sigma, \Sigma^{\prime}$. It then follows that

$$
Z^{\mathrm{d}}=\mu+R U^{(2)} A, Z^{\prime} \stackrel{d}{=} \mu+R U^{(2)} A^{\prime}
$$

When one, but not both, of $\rho, \rho^{\prime}$ equals 1 in absolute value, say $-1<\rho^{\prime}<\rho=1$, then $Z^{\prime} \stackrel{\mathrm{d}}{=} \mu+\mathrm{RU}{ }^{(2)} \mathrm{A}^{\prime}$ and $E \mathrm{e}^{\mathrm{is}\left(Z^{\prime}-\mu\right)^{t}}=\phi\left(s \Sigma^{\prime} s^{t}\right)=\int_{[0, \infty)} \Omega_{2}\left(r^{2} s \Sigma^{\prime} s^{t}\right) \mathrm{dF}(r)$ where $F$ is a distribution of $R$. Since

$$
E e^{i s(z-\mu)^{t}}=\phi\left(s \Sigma s^{t}\right)=\int_{[0, \infty)} \Omega_{2}\left(r^{2} s \Sigma s^{t}\right) d F(r)
$$

it is easily checked that $\mathrm{z} \stackrel{\mathrm{d}}{=} \mu+\mathrm{RU}^{(2)} \mathrm{A}$. Hence (3.2) holds provided at least one of $\rho, \rho^{\prime}$ differs from 1 in absolute value. Because of the independence of $R, U^{(2)}$, arguing as before, it suffices to show that $E l\left(U^{(2)} A\right) \geq E k\left(U^{(2)} A^{\prime}\right)$. This will be done by defining a random vector $\left(X_{1}, X_{2}, Y_{1}, Y_{2}\right)$ which satisfies condition ( $C O)$; $\left(X_{1}, Y_{1}\right)$ and $\left(X_{2}, Y_{2}\right)$ will be distributed as $U^{(2)} A_{,}\left(X_{1}, Y_{2}\right)$ and $\left(X_{2}, Y_{1}\right)$ will be distributed as $U^{(2)} A^{\prime}$, and the product $\left(X_{2}-X_{1}\right)\left(Y_{2}-Y_{1}\right)$ will be nonnegative.

The random vector $U^{(2)}$ can be taken to be $(\sin \theta, \cos \theta)$, where $\theta$ is uniformly distributed on any interval of length $2 \pi$. Then

$$
U^{(2)} A=\left(\sigma_{1} \sin (\theta+\alpha), \sigma_{2} \cos (\theta-\alpha)\right), U^{(2)} A^{\prime}=\left(\sigma_{1} \sin \left(\theta+\alpha^{\prime}\right), \sigma_{2} \cos \left(\theta-\alpha^{\prime}\right)\right) .
$$


Let

$$
S=\sin (\theta+\alpha), T=\cos (\theta-\alpha), V=\sin \left(\alpha^{\prime}-\theta\right),
$$

and define

$$
X_{1}=\sigma_{1} S, X_{2}=\sigma_{1}\left(S+2 \cos \left(\alpha+\alpha^{\prime}\right) V\right), Y_{1}=\sigma_{2} T, Y_{2}=\sigma_{2}\left(T+2 \sin \left(\alpha-\alpha^{\prime}\right) V\right)
$$

Since $2 \sin \left(\alpha-\alpha^{\prime}\right) \cos \left(\alpha+\alpha^{\prime}\right)=\sin 2 \alpha-\sin 2 \alpha^{\prime}=\rho-\rho^{\prime} \geq 0$, it follows that $\left(X_{2}-X_{1}\right)\left(Y_{2}-Y_{1}\right) \geq 0$. By further trigonometric manipulations, one obtains

$$
S+2 \cos \left(\alpha+\alpha^{\prime}\right) V=\sin \left(\left(2 \alpha^{\prime}-\theta\right)+\alpha\right), T+2 \sin \left(\alpha-\alpha^{\prime}\right) V=\cos \left(\left(2 \alpha^{\prime}-\theta\right)-\alpha\right)
$$

Since $2 \alpha^{\prime}-\theta$ is uniformly distributed on an interval of 1 ength $2 \pi,\left(X_{2}, Y_{2}\right)$, as well as $\left(X_{1}, Y_{1}\right)$, is distributed as $U^{(2)} A$. Similarly, one finds that $\left(X_{1}, Y_{2}\right)$ and $\left(\mathrm{X}_{2}, \mathrm{Y}_{1}\right)$ are distributed as $\mathrm{U}^{(2)} \mathrm{A}^{\prime}$. This completes the proof.

Remark. Implicit in this proof is the use of a fact about any two ellipses which are inscribed in the same rectangle. Each point on one of the ellipses is the vertex of a rectangle whose opposite vertex is on the same ellipse and whose adjacent vertices are on the other ellipse. (In fact there are two such rectangles.) Whether this is a known fact from projective geometry is unknown to the authors. (In the present context, the two ellipses are the ranges of the random vectors $\mathrm{U}^{(2)} \mathrm{A}$ and $\mathrm{U}^{(2)} \mathrm{A}^{\prime}$.)

When $\mathrm{k}$ and $\ell$ are functions of $\mathrm{x} \pm \mathrm{y}$.

Suppose $k$ and $l$, which are defined for $(x, y) \in \mathbb{R}^{2}$, are functions of the sum $x+y$. For convenience, we shall write them as $k(x+y)$ and $\ell(x+y)$. In terms of 


$$
u=\frac{1}{2}\left(x_{1}+x_{2}+y_{1}+y_{2}\right), v=\frac{1}{2}\left(x_{2}-x_{1}+y_{2}-y_{1}\right), w=\frac{1}{2}\left|x_{2}-x_{1}+y_{1}-y_{2}\right|,
$$

the inequalities (1.15) become

$\ell(u+v)+\ell(u-v) \geq k(u+w)+k(u-w), \quad u \in \mathbb{R}, \quad 0 \leq w \leq v<\infty$.

When $k=l$, this condition is equivalent to convexity. Thus $k(x+y)$ is quasi-monotone (as a function of $(x, y)$ ) if and only if it is a convex function (of the single variable $t=x+y$ ).

In general, $\mathrm{k}$ can be no larger than $\mathrm{k}^{*}$ defined by

$$
k^{*}(u)=\frac{1}{2} \inf _{v \geq 0}\{\ell(u+v)+\ell(u-v)\}
$$

(Set $\mathrm{w}=0$ in (3.4).) Assuming that $\mathrm{k}^{*}$ is finite and measurable, $\mathrm{k}$ can equal $\mathrm{k}^{*}$ if and only if

$$
\inf _{\alpha \geq 0}\{\ell(u+w+\alpha)+\ell(u+w-\alpha)\}+\inf _{\alpha \geq 0}\{\ell(u-w+\alpha)+\ell(u-w-\alpha)\} \leq 2\{\ell(u+v)+\ell(u-v)\}
$$

for all $u \in \mathbb{R}, 0 \leq w \leq v<\infty$. This condition is met if for each $u \in \mathbb{R}, w \geq 0$ and $v \geq w$, there exists an $\alpha \geq 0$ such that

$$
f_{u}(w+\alpha)+f_{u}(w-\alpha) \leq 2 f_{u}(v)
$$

where $f_{u}$ is the even function defined by $f_{u}(v)=\ell(u+v)+\ell(u-v)$. A suitable value for $\alpha$ can be obtained if $f_{u}$ is nondecreasing (set $\alpha=0$ ), nonincreasing (set $\alpha=v+w)$, or concave (set $\alpha=v$ ) on $[0, \infty)$. Summarizing, if $k^{*}$ is finite and measurable, and if for each $u, l(u+v)+\ell(u-v)$ is a nondecreasing, nonincreasing or concave function of $v$ on $[0, \infty)$, then $k^{*}$ is a suitable and maximal choice for $k$. Under these conditions on $k^{*}$ and $l$, any measurable function $k \leq k$ satisfies (3.4). 
The following are examples of functions $l$ which meet these conditions:

(i) If $l$ is convex (as a function of the single variable $t=x+y$ ), then $\ell(u+v)+\ell(u-v)$ is a nondecreasing function of $v$ on $[0, \infty)$ and $k^{*}=\ell$.

(ii) If $l$ is a symmetric function with respect to some point $\left(u_{0}, l_{0}\right)$ in $\mathbb{R}^{2}$ (i.e., if $\left.\ell\left(u_{0}+v\right)+\ell\left(u_{0}-v\right)=2 \ell_{0}, v \geq 0\right)$, and $\ell$ is concave on $\left[u_{0}, \infty\right)$, then $\ell(u+v)+\ell(u-v)$ is a nonincreasing function of $v \geq 0$ for $u \geq u_{0}$ and is a nondecreasing function of $v \geq 0$ for $u \leq u_{0}$. It follows that $k^{*}=\ell$ on $\left(-\infty, u_{0}\right]$.

The values of $k^{*}$ on $\left(u_{0}, \infty\right)$ must be evaluated from (3.5) in each specific case, and it must be checked whether $k^{*}$ is finite and measurable. For instance, if $\ell$ is the distribution function of a normal random variable with mean $\mu$ and variance $\sigma^{2}$, then $k^{*}=\ell$ on $(-\infty, \mu]$ and $k^{*}=\frac{1}{2}$ on $[\mu, \infty)$; hence $k^{*}$ is a maximal choice for $k$. (This is another example of functions $k$ and $\ell$ satisfying (1.15) which can not be separated by a quasi-monotone function $\mathrm{m}$, i.e., there is no function $m$ which satisfies (1.14) and $k \leq m \leq l$. The details are left to the reader.)

When the supports of $X+Y$ and $X^{\prime}+Y^{\prime}$ are not the entire real line, the ranges of $u, v$ and $w$ in $(3.6)$ can be reduced. Thus $k$ and $l$ will have to satisfy fewer restrictions, and a wider variety of suitable $k$, \& pairs will be permitted. (The same can be said when $k$ and $l$ are general functions on $\mathbb{R}^{2}$ and the supports of $(X, Y)$ and $\left(X^{\prime}, Y^{\prime}\right)$ are proper subsets of $\mathbb{R}^{2}$.)

When $k$ and $l$ are functions of $x-y$ (instead of $x+y$ ) the analogue of (3.4) is

$\ell(u+w)+\ell(u-w) \geq k(u+v)+k(u-v), \quad u \in \mathbb{R}, \quad 0 \leq w \leq v<\infty$,

(the definitions of $u, v$ and $w$ must be modified appropriately) and the analysis of (3.7) is similar to that given for (3.4). 
A generalization to higher dimensions.

A higher dimensional version of Theorem 3.2 can be proven by reducing the dimension to 2 through conditioning.

Theorem 3.3. Suppose that $Z \sim \mathrm{EC}_{\mathrm{n}}(\mu, \Sigma, \phi)$ and $\mathrm{Z}^{\prime} \sim \mathrm{EC}_{\mathrm{n}}\left(\mu, \Sigma^{\prime}, \phi\right)$ where $\Sigma=\left(\sigma_{i j}\right), \Sigma^{\prime}=\left(\sigma_{i j}^{\prime}\right), \sigma_{i i}=\sigma_{i i}^{\prime}, \sigma_{i j} \geq \sigma_{i j}^{\prime}, i \neq j$. Then (1.3) holds for any pair of functions $k$ and $l$ of $n$ variables for which the expectations appearing in (1.3) make sense and which satisfy (1.15) as functions of any two of the $\mathrm{n}$ variables for all fixed values of the remaining $n-2$ variables.

Proof. According to the argument in the first paragraph of the proof of Theorem 5.1 in [3], it suffices to prove the result in the case where $\sigma_{12}>\sigma_{12}^{\prime}$ and $\sigma_{i j}=\sigma_{i j}^{\prime}$ for all $(i, j) \neq(1,2),(2,1)$. Write

$$
z=\left(z_{1}, z_{2}\right), \mu=\left(\mu_{1}, \mu_{2}\right), \Sigma=\left(\begin{array}{ll}
\Sigma_{11} & \Sigma_{12} \\
\Sigma_{21} & \Sigma_{22}
\end{array}\right)
$$

where $Z_{1}, \mu_{1}$ are two-dimensional and $\Sigma_{11}$ is $2 \times 2$. It is easily seen via the characteristic function that

$$
\left(Y_{1}, Y_{2}\right)=\left(Z_{1}-\mu_{1}, Z_{2}-\mu_{2}\right)\left(\begin{array}{cc}
I & 0 \\
-\Sigma_{22}^{+} \Sigma_{21} & I
\end{array}\right) \sim \mathrm{EC}_{n}\left(0,\left(\begin{array}{ll}
\Sigma^{*} & 0 \\
0 & \Sigma_{22}
\end{array}\right), \phi\right)
$$

where $\Sigma_{22}^{+}$is the self-adjoint generalized inverse of $\Sigma_{22}$ and $\Sigma^{*}=\Sigma_{11}-\Sigma_{12} \Sigma_{22}^{+} \Sigma_{21}$. Now let $\left.\mathrm{RU}^{(\mathrm{n})}=\mathrm{R} \mathrm{U}_{1}, \mathrm{U}_{2}\right) \sim \mathrm{EC}_{\mathrm{n}}(0, \mathrm{I}, \phi)$, where $\mathrm{U}_{1}$ is two-dimensional. Then

$$
\left(Y_{1}, Y_{2}\right) \stackrel{\mathrm{d}}{=} \mathrm{R}\left(\mathrm{U}_{1}, \mathrm{U}_{2}\right)\left(\begin{array}{ll}
\Sigma^{*} & 0 \\
0 & \Sigma_{22}
\end{array}\right)^{\frac{1}{2}}=\mathrm{R}\left(\mathrm{U}_{1} \Sigma^{*^{\frac{1}{2}}}, \mathrm{U}_{2} \Sigma_{22}^{\frac{1}{2}}\right)
$$

and 


$$
\mathrm{z}=\left(\mathrm{z}_{1}, \mathrm{z}_{2}\right) \stackrel{\mathrm{d}}{=}\left(\mu_{1}+\mathrm{RU} \mathrm{L}_{2} \Sigma_{22}^{\frac{1}{2}} \Sigma_{22}^{+} \Sigma_{12}+\mathrm{RU}_{1} \Sigma^{*^{\frac{1}{2}}}, \mu_{2}+\mathrm{RU} \mathrm{L}_{22}^{\frac{1}{2}}\right)
$$

Since $U^{(n)}$ is uniformly distributed on the surface of the n-dimensional unit sphere, $\left(\mathrm{U}_{1} \mid \mathrm{U}_{2}=\mathrm{u}_{2}\right) \stackrel{\mathrm{d}}{=}\left[1-\mathrm{u}_{2} \mathrm{u}_{2}^{\mathrm{t}_{2}}\right]_{+}^{\frac{1}{2}} \mathrm{U}^{(2)}$ where $[\mathrm{a}]_{+}=\max (\mathrm{a}, 0)$. (See for instance Lemma 3 in [1].) Since $R$ and $V$ are independent, it follows that for all $r \geq 0$ and $\mathrm{u}_{2}$,

$$
\left(\left(z_{1}, z_{2}\right) \mid R=r, U_{2}=u_{2}\right) \stackrel{d}{=}\left(\mu^{*}+r^{*} U^{(2)} \sum^{* \frac{1}{2}}, \mu_{2}+r u_{2} \Sigma_{22}^{\frac{1}{2}}\right)
$$

where

$$
\mu^{*}=\mu_{1}+r u_{2} \Sigma_{22}^{\frac{1}{2}} \Sigma_{22}^{+} \Sigma_{12}, r^{*}=x\left[1-u_{2} u_{2}^{t}\right]_{+}^{\frac{1}{2}}, \Sigma^{*}=\Sigma_{11}-\Sigma_{12} \Sigma_{22}^{+} \Sigma_{21}
$$

Since $\Sigma^{\prime}=\left(\begin{array}{cc}\Sigma_{11}^{\prime} & \Sigma_{12} \\ \Sigma_{21} & \Sigma_{22}\end{array}\right), z^{\prime}=\left(z_{1}^{\prime}, z_{2}^{\prime}\right)$ satisfies $(3.8)$ with $\mu^{* \prime}=\mu^{*}, r^{*}=r^{*}$ and $\Sigma^{* \prime}=\Sigma_{11}^{\prime}-\Sigma_{12} \Sigma_{22}^{+} \Sigma_{21}$. In order to verify $E \ell(Z) \geq E k\left(Z^{\prime}\right)$ it thus suffices to show that for al1 $\mathrm{r} \geq 0$ and $\mathrm{u}_{2}$,

$$
E \ell\left(\mu^{*}+r^{*} U^{(2)} \Sigma^{* \frac{1}{2}}, \mu_{2}+r u_{2} \Sigma_{22}^{\frac{1}{2}}\right) \geq E k\left(\mu^{*}+r^{*} U^{(2)} \Sigma^{* 1^{1 / 2}}, \mu_{2}+r u_{2} \Sigma_{22}^{\frac{1}{2}}\right)
$$

and this follows from Theorem 3.2.

Remarks. 1. By letting $\mathrm{k}$ and $\ell$ in Theorem 3.3 be indicator functions of $(-\infty, z], z \in \mathbb{R}^{n}$, one obtains the inequality $H \geq H^{\prime}$ where $H$ and $H^{\prime}$ are the distribution functions of $z$ and $z$ ' respectively. For normal distributions this inequality is a wel1-known result due to Slepian [9], and for elliptically contoured distributions it has been obtained by Das Gupta et al. [3] under the assumptions that the matrices $\Sigma$ are invertible and that densities exist. 
2. Under the assumptions of Theorem 3.3 it is also true that $P(Z \in A) \geq P\left(Z^{\prime} \in A\right)$ for every set of the form $\left\{u \in \mathbb{R}^{n}, u \geq z\right\}, z \in \mathbb{R}^{n}$. Thus all results in this section are concerned with random vectors that are stochastically ordered in the sense described in the beginning of this section. (This observation may point the way to extensions that are not confined to Euclidean-space-valued random variables.)

3. The approach used in proving Theorem 3.3 can be used to extend the theorem to random vectors $Z$ and $Z$ ' with more general distributions than elliptically contoured. For instance the theorem holds for random variables with distributions $z^{\stackrel{d}{=}} \mu+U^{(k)} A R$ and $Z^{\prime} \stackrel{d}{=} \mu+U^{(k)} A^{\prime} R$, where $R$ is any random matrix with nonnegative components which is independent of $U^{(k)}$.

4. Necessary and sufficient conditions for (1.3)

It is possible to characterize the bivariate distribution functions $H$ and $H^{\prime}$ which satisfy condition $(\mathrm{C} 0)$. This is accomplished by a straightforward generalization of the proof given by Sudakov [11] of a theorem by Strassen [10] Cast in our context this slight extension of Strassen's theorem says that (C0) is equivalent to the following condition (C1):

$$
P(Z \in A) \geq P\left(Z^{\prime} \in A^{\prime}\right), \quad\left(A, A^{\prime}\right) \in A_{2}=\left\{\left(A, A^{\prime}\right): 1_{A}, 1_{A^{\prime}} \text {, satisfy (1.15) }\right\}
$$

Thus if (C3) denotes the following,

$$
E l(Z) \geq E k\left(Z^{\prime}\right)
$$

$(l, k) \in F_{2}$, the class of all functions $l, k$ which satisfy (1.15) and for which the expectations are defined,

we have that conditions (C0), (C1) and (C3) are equivazent. The implications $(\mathrm{CO}) \Rightarrow(\mathrm{C} 3)$ and $(\mathrm{C} 3) \Rightarrow(\mathrm{C} 1)$ are immediate, and the nontrivial ones are $(\mathrm{C} 1) \Rightarrow(\mathrm{C} 3) \Rightarrow(\mathrm{C} 0)$. 
Two indicator functions $k=1_{A}$ and $\ell=1_{B}$ satisfy (1.15) if and only if $A \subseteq B$ and for every rectangular set of four points in $\mathbb{R}^{2}$

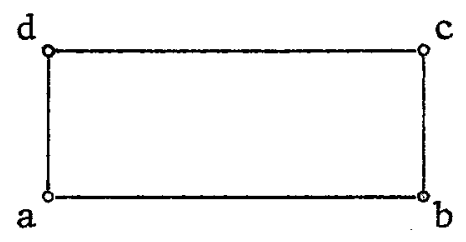

the number of points of $\{a, c\}$ in $B$ is larger than or equal to the number of points of $\{b, d\}$ in $A$. By choosing various such pairs of sets $A$ and $B$ one finds that the inequality in (C3) requires $H$ and $H^{\prime}$ to have common marginal distributions and to satisfy $H^{\prime} \geq H^{\prime}$. In the case of normal or elliptically contoured distributions $\mathrm{H}$ and $\mathrm{H}^{\prime}$, these two properties imply (C3) as shown in Theorems 3.1 and 3.2. The case of more general distributions requires further investigation.

Because of the equivalence of conditions (C0), (C1) and (C3), our goal, i.e. condition ( $\mathrm{C} 3$ ), can be achieved by establishing either condition (CO) or (C1). In Theorems 3.1 and 3.2 condition (CO) is established. Even though condition (CI) is very natural and satisfactory, especially in its relationship with (C3), it is unfortunately very difficult to verify, and we have failed to achieve this for any distributions $H_{\text {and }} \mathrm{H}^{\prime}$.

The equivalence of ( $\mathrm{C} 0$ ), (C1) and ( $\mathrm{C} 3$ ) is a special case of a more general situation which provides new ways of obtaining inequalities of the type (1.3), and of showing the existence of joint distributions with fixed support and with certain marginals fixed. To illustrate the power and novelty of this approach let us consider a few examples.

Let $F$ be a closed subset of $\mathbb{R}^{4}$, and consider an inequality between functions $k$ and $\ell$ of the following type (simpler than (1.15)) 
(CF)

$$
\ell\left(x_{1}, y_{1}\right) \geq k\left(x_{2}, y_{2}\right), \quad\left(x_{1}, x_{2}, y_{1}, y_{2}\right) \in F,
$$

and the following conditions which depend on F.

(COF) There exists a random vector $\left(\mathrm{X}_{1}, \mathrm{X}_{2}, \mathrm{Y}_{1}, \mathrm{Y}_{2}\right)$ whose values are in the set $F$ and which is such that the bivariate marginal distributions of $\left(X_{1}, Y_{1}\right)$ and $\left(\mathrm{X}_{2}, \mathrm{Y}_{2}\right)$ are $\mathrm{H}$ and $\mathrm{H}^{\prime}$ respectively.

(C1F) $P(Z \in A) \geq P\left(Z^{\prime} \in A^{\prime}\right)$,

$\left(A, A^{\prime}\right) \in A_{2}=\left\{\left(A, A^{\prime}\right): 1_{A},{ }_{A}\right.$, satisfy $\left.(C F)\right\}$.

(C3F) $E \ell(Z) \geq E k\left(Z^{\prime}\right)$,

$(l, k) \in F_{2}$, the class of all functions $l, k$ which satisfy (CF) and for which the expectations are defined.

By Strassen's theorem, (COF) and (C1F) are equivalent. Also, if (COF) holds we have

$$
\ell\left(X_{1}, Y_{1}\right) \geq k\left(X_{2}, Y_{2}\right) \text { a.s. }
$$

for all pairs $k$ and $\ell$ satisfying (CF), and by taking expectations (C3F) follows. Thus $(\mathrm{COF}) \Rightarrow(\mathrm{C} 3 \mathrm{~F})$ and clearly $(\mathrm{C} 3 \mathrm{~F}) \Rightarrow(\mathrm{C} 1 \mathrm{~F})$. Hence conditions $(\mathrm{COF})$, (C1F) and $(\mathrm{C} 3 \mathrm{~F})$ are equivalent. By choosing

$$
F=\left\{\left(x_{1}, x_{2}, y_{1}, y_{2}\right): x_{1} \geq x_{2}, y_{1} \geq y_{2}\right\} \text {, }
$$

(CF) becomes $\ell\left(x_{1}, y_{1}\right) \geq k\left(x_{2}, y_{2}\right), x_{1} \geq x_{2}, y_{1} \geq y_{2}$; (C1F) is equivalent to

$$
H(I) \geq H^{\prime}(I) \text { for all increasing sets } I \text {; }
$$

and the result includes Theorem 1 (i), (iv), and (vi) of Kamae, Krengel and 0 Brien [4]. Of course, any one or both of the inequalities in the definition of $\mathrm{F}$ could be reversed with corresponding results. If we choose 


$$
F=\left\{\left(x_{1}, x_{2}, y_{1}, y_{2}\right): \max \left(\left|x_{1}\right|,\left|y_{1}\right|\right) \leq \max \left(\left|x_{2}\right|,\left|y_{2}\right|\right)\right\},
$$

then $(\mathrm{C} 1 \mathrm{~F})$ becomes equivalent to

$$
H(A) \geq H^{\prime}(A) \text { for all squares } A=\{(x, y):|x| \leq a,|y| \leq a\} \text {, }
$$

and thus (4.1), (COF) and (C3F) are equivalent. When $H$ and $H^{\prime}$ are normal with zero means, common variances and correlation coefficients $\rho$ and $\rho^{\prime}$ satisfying $|\rho| \geq\left|\rho^{\prime}\right|$, then (4.1) is satisfied and thus (COF) and (C3F) hold, both new results. The same is true for absolutely continuous elliptically contoured distributions $\mathrm{EC}_{2}(0, \Sigma, \phi)$ and $\mathrm{EC}_{2}\left(0, \Sigma^{\prime}, \phi\right)$, where $\Sigma, \Sigma^{\prime}$ are as in Theorem 3.2 with $|\rho| \geq\left|\rho^{\prime}\right|$; in this case inequality (4.1) follows from Theorem 2.1 of [3]

For not necessarily absolutely continuous elliptically contoured distributions $\mathrm{EC}_{2}(0, \Sigma, \phi)$ and $\mathrm{EC}_{2}\left(0, \Sigma^{\prime}, \phi\right)$, where $\Sigma, \Sigma^{\prime}$ are as in Theorem 3.1 with $|\rho| \geq\left|\rho^{\prime}\right|$, we now give a simple proof of (C.3F), and thus also of (4.1), in the case where the common variances of $\Sigma, \Sigma^{\prime}$ are equal. The approach is through a construction similar to that of Theorem 3.2 and thus the result is obtained without using Strassen's theorem. Also the result is slightly stronger than that in the previous paragraph in that the elliptically contoured distributions are not required to be absolutely continuous. Even though we are assuming for simplicity of the construction that the common variances of $\Sigma, \Sigma^{\prime}$ are equal, no. doubt a similar, but somewhat more involved, construction would be feasible when the variances are not equal.

Theorem 4.1. Suppose that $\mathrm{Z} \sim \mathrm{EC}_{2}(0, \Sigma, \phi)$ and $\mathrm{Z}^{\prime} \sim \mathrm{EC}_{2}\left(0, \Sigma^{\prime}, \phi\right)$ where

$$
\Sigma=\left(\begin{array}{cc}
\sigma^{2} & \rho \sigma^{2} \\
\rho \sigma^{2} & \sigma^{2}
\end{array}\right), \Sigma^{\prime}=\left(\begin{array}{cc}
\sigma^{2} & \rho^{\prime} \sigma^{2} \\
\rho^{\prime} \sigma^{2} & \sigma^{2}
\end{array}\right)
$$

and $|\rho| \geq\left|\rho^{\prime}\right|$. Then (C3F) holds, as welz as (4.1). 
Proof. As in the proof of Theorem 3.2 it suffices to show $E l\left(U^{(2)} A\right) \geq E k\left(U^{(2)} A^{\prime}\right)$ (here $\mu=0)$. This will be done by defining a random vector $\left(X_{1}, X_{2}, Y_{1}, Y_{2}\right)$ which satisfies condition (COF); i.e. $\left(X_{1}, Y_{1}\right) \stackrel{d}{=} U^{(2)} A,\left(X_{2}, Y_{2}\right) \stackrel{d}{=} U^{\prime}{ }^{(2)} A^{\prime}$, and $\max \left(\left|\mathrm{X}_{1}\right|,\left|\mathrm{Y}_{1}\right|\right) \leq \max \left(\left|\mathrm{X}_{2}\right|,\left|\mathrm{Y}_{2}\right|\right)$.

The random vectors $U^{(2)}$ and $U^{\prime}(2)$ can be taken to be $(\cos \theta, \sin \theta)$ and $\left(\cos \theta^{\prime}, \sin \theta^{\prime}\right)$, where $\theta$ and $\theta^{\prime}$ are uniformly distributed on intervals of length $2 \pi$. Then we can take

$$
\left(X_{1}, Y_{1}\right)=\sigma(\cos (\theta-\alpha), \sin (\theta+\alpha)),\left(X_{2}, Y_{2}\right)=\sigma\left(\cos \left(\theta^{\prime}-\alpha^{\prime}\right), \sin \left(\theta^{\prime}+\alpha^{\prime}\right)\right)
$$

where $\sin 2 \alpha=\rho, \sin 2 \alpha^{\prime}=\rho^{\prime},-\frac{\pi}{4} \leq \alpha, \alpha^{\prime} \leq \frac{\pi}{4}$. We will now determine the joint distribution of $\left(\theta, \theta^{\prime}\right)$ so that the marginals will be uniform on intervals of length $2 \pi$ and

$$
\max \{|\cos (\theta-\alpha)|,|\sin (\theta+\alpha)|\} \leq \max \left\{\left|\cos \left(\theta^{\prime}-\alpha^{\prime}\right)\right|,\left|\sin \left(\theta^{\prime}+\alpha^{\prime}\right)\right|\right\} .
$$

We have

$$
\begin{aligned}
\left|\cos \left(\theta^{\prime}-\alpha^{\prime}\right)\right| \geq\left|\sin \left(\theta^{\prime}+\alpha^{\prime}\right)\right| & \Leftrightarrow \cos 2\left(\theta^{\prime}-\alpha^{\prime}\right) \geq-\cos 2\left(\theta^{\prime}+\alpha^{\prime}\right) \\
& \Leftrightarrow \cos 2 \theta^{\prime} \cos 2 \alpha^{\prime} \geq 0 \Leftrightarrow \cos 2 \theta^{\prime} \geq 0
\end{aligned}
$$

and similarly

$$
\begin{aligned}
& |\cos (\theta-\alpha)| \leq\left|\cos \left(\theta^{\prime}-\alpha^{\prime}\right)\right| \Leftrightarrow \sin \left(\theta+\theta^{\prime}-\gamma\right) \sin \left(\theta-\theta^{\prime}-\beta\right) \geq 0 \\
& |\sin (\theta+\alpha)| \leq\left|\cos \left(\theta^{\prime}-\alpha^{\prime}\right)\right| \Leftrightarrow \cos \left(\theta+\theta^{\prime}+\beta\right) \cos \left(\theta-\theta^{\prime}+\gamma\right) \geq 0 \\
& |\cos (\theta-\alpha)| \leq\left|\sin \left(\theta^{\prime}+\alpha^{\prime}\right)\right| \Leftrightarrow \cos \left(\theta+\theta^{\prime}-\beta\right) \cos \left(\theta-\theta^{\prime}-\gamma\right) \leq 0 \\
& |\sin (\theta+\alpha)| \leq\left|\sin \left(\theta^{\prime}+\alpha^{\prime}\right)\right| \Leftrightarrow \sin \left(\theta+\theta^{\prime}+\gamma\right) \sin \left(\theta-\theta^{\prime}+\beta\right) \leq 0
\end{aligned}
$$

where $\beta=\alpha-\alpha^{\prime}, \gamma=\alpha+\alpha^{\prime}\left(0 \leq \beta \leq \frac{\pi}{4}, 0 \leq \gamma \leq \frac{\pi}{2}\right)$. Thus (4.2) is equivalent to 


$$
\left\{\begin{array}{l}
\cos 2 \theta^{\prime} \geq 0 \\
\sin \left(\theta+\theta^{\prime}-\gamma\right) \sin \left(\theta-\theta^{\prime}-\beta\right) \geq 0 \\
\cos \left(\theta+\theta^{\prime}+\beta\right) \cos \left(\theta-\theta^{\prime}+\gamma\right) \geq 0
\end{array}\right\} \text { or }\left\{\begin{array}{l}
\cos 2 \theta^{\prime} \leq 0 \\
\cos \left(\theta+\theta^{\prime}-\beta\right) \cos \left(\theta-\theta^{\prime}-\gamma\right) \leq 0 \\
\sin \left(\theta+\theta^{\prime}+\gamma\right) \sin \left(\theta-\theta^{\prime}+\beta\right) \leq 0
\end{array}\right\} \text {. }
$$

The two sets of inequalities in (4.3) determine the set where the support of the joint distribution of $\left(\theta, \theta^{\prime}\right)$ must lie.

Let us first consider the case where $0 \leq \rho^{\prime} \leq \rho$, i.e. $0 \leq \alpha^{\prime} \leq \alpha \leq \frac{\pi}{4}$. When $\alpha=\alpha^{\prime}$, i.e. $\beta=0$, we can take $\theta=\theta^{\prime}$. In the general case, $\beta>0$, we can take $\theta$ to be the following function of $\theta^{\prime}$ :

$$
\begin{aligned}
\theta & =\theta^{\prime}+\beta \quad \text { for }-\frac{\pi}{4} \leq \theta^{\prime}<\frac{\pi}{4}-\beta, & & \frac{3 \pi}{4} \leq \theta^{\prime}<\frac{5 \pi}{4}-\beta \\
& =\theta^{\prime}+\beta-\frac{\pi}{2} \text { for } \frac{\pi}{4}-\beta \leq \theta^{\prime}<\frac{\pi}{4}, & & \frac{5 \pi}{4}-\beta \leq \theta^{\prime}<\frac{5 \pi}{4} \\
& =\theta^{\prime}-\beta \quad \text { for } \frac{\pi}{4}+\beta \leq \theta^{\prime}<\frac{3 \pi}{4}, & & \frac{5 \pi}{4}+\beta \leq \theta^{\prime}<\frac{7 \pi}{4} \\
& =\theta^{\prime}-\beta+\frac{\pi}{2} \text { for } \frac{\pi}{4} \leq \theta^{\prime}<\frac{\pi}{4}+\beta, & & \frac{5 \pi}{4} \leq \theta^{\prime}<\frac{5 \pi}{4}+\beta .
\end{aligned}
$$

Since the relationship between $\theta$ and $\theta^{\prime}$ in $\left[-\frac{\pi}{4}, \frac{7 \pi}{4}\right)$ is one-to-one and piecewise linear with slope 1 , if $\theta^{\prime}$ is uniform $1 y$ distributed on $\left[-\frac{\pi}{4}, \frac{7 \pi}{4}\right]$, then so is $\theta$. Moreover the pairs $\left(\theta, \theta^{\prime}\right)$ defined by (4.4) satisfy conditions (4.3) and we now check this for $-\frac{\pi}{4} \leq \theta^{\prime}<\frac{\pi}{4}$, in which case $\cos 2 \theta^{\prime} \geq 0$ (the remaining cases being similar). When $-\frac{\pi}{4} \leq \theta^{\prime}<\frac{\pi}{4}-\beta$, we have $\theta=\theta^{\prime}+\beta$ and thus

$$
\sin \left(\theta+\theta^{\prime}-\gamma\right) \sin \left(\theta-\theta^{\prime}-\beta\right)=0 \geq 0, \cos \left(\theta+\theta^{\prime}+\beta\right) \cos \left(\theta-\theta^{\prime}+\gamma\right)=\cos 2 \theta \cos 2 \alpha \geq 0 .
$$

When $\frac{\pi}{4}-\beta \leq \theta^{\prime}<\frac{\pi}{4}$ we have $\theta=\theta^{\prime}+\beta-\frac{\pi}{2}$ and thus

$$
\sin \left(\theta+\theta^{\prime}-\gamma\right) \sin \left(\theta-\theta^{\prime}-\beta\right)=\sin \left(2 \theta^{\prime}+\beta-\frac{\pi}{2}-\gamma\right) \sin \left(-\frac{\pi}{2}\right)=\cos 2\left(\theta^{\prime}-\alpha^{\prime}\right) \geq 0
$$


since $0 \leq \theta^{\prime}-\alpha^{\prime} \leq \frac{\pi}{4}$, and

$$
\cos \left(\theta+\theta^{\prime}+\beta\right) \cos \left(\theta-\theta^{\prime}+\gamma\right)=\cos \left(2 \theta+\frac{\pi}{2}\right) \cos \left(\beta+\gamma-\frac{\pi}{2}\right)=-\sin 2 \theta \sin 2 \alpha \geq 0
$$

since $-\frac{\pi}{4} \leq \theta<-\frac{\pi}{8}$.

The remaining cases are treated similarly, and Figure II shows the graph of $\theta=f\left(\theta^{\prime}\right)$ which achieves the desired properties. The graph is plotted for $-\frac{\pi}{4} \leq \theta^{\prime}<\frac{3 \pi}{4}$, and for $\frac{3 \pi}{4} \leq \theta<\frac{7 \pi}{4}$ the graph is obtained by shifting the plotted graph by $(\pi, \pi)$.

\section{If we choose}

$$
F=\left\{\left(x_{1}, x_{2}, y_{1}, y_{2}\right):\left|x_{1}\right| \geq\left|x_{2}\right| \text { or }\left|y_{1}\right| \geq\left|y_{2}\right|\right\}
$$

then (C1F) becomes equivalent to

$H\left(A^{c}\right) \geq H^{\prime}\left(A^{c}\right)$, i.e. $H(A) \leq H^{\prime}(A)$, for all rectangles $A=\{(x, y):|x| \leq a,|y| \leq b\}$,

and the corresponding conditions (COF), (C3F) and (4.5) are equivalent. When $\mathrm{H}$ and $\mathrm{H}^{\prime}$ are absolutely continuous elliptically contoured distributions $\mathrm{EC}_{2}(0, \Sigma, \phi)$ and $\mathrm{EC}_{2}\left(0, \Sigma^{\prime}, \phi\right)$, where $\Sigma, \Sigma^{\prime}$ are as in Theorem 3.2 with $|\rho| \leq\left|\rho^{\prime}\right|$, then (4.5) is Theorem 2.1 of [3], and thus (COF) and (C3F) both hold. If we choose

$$
F=\left\{\left(x_{1}, x_{2}, y_{1}, y_{2}\right):\left(x_{1}, y_{1}\right) \geq^{m}\left(x_{2}, y_{2}\right)\right\}
$$

where for two-dimensional vectors $\left(x_{1}, y_{1}\right) \geq\left(x_{2}, y_{2}\right)$ means $\max \left(x_{1}, y_{1}\right) \geq \max \left(x_{2}, y_{2}\right)$ and $x_{1}+y_{1}=x_{2}+y_{2}$, then (C1F) becomes equivalent to 


$$
H(A) \geq H^{\prime}(A) \text { for all measurable Schur-convex sets } A
$$

(A is Schur-convex if $Z \in A$ and $Z^{\prime} z^{m} Z$ imply $Z^{\prime} \in A$ ) and the corresponding conditions (COF), (C3F) and (4.6) are equivalent. This is Theorem 2.2 in [6]. A11 the examples described above have obvious n-dimensional analogues. 

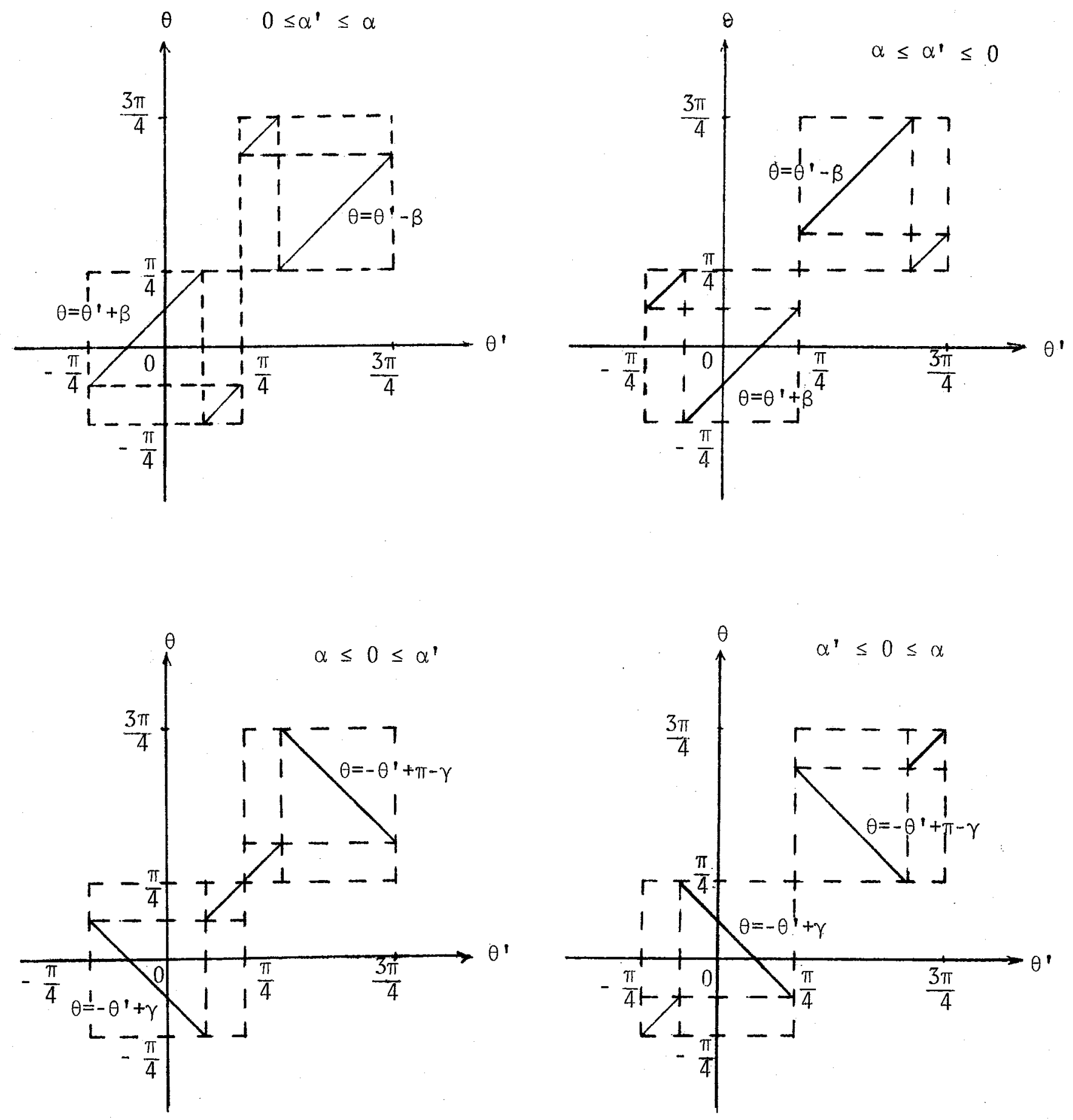


\section{References}

1. Cambanis, S., Huang, S., Simons, G.: On the theory of elliptically contoured distributions. Institute of Statistics Mimeo Series No. 1246. University of North Carolina at Chape1 Hil1 (1979) .

2. Cambanis, S., Simons, G., Stout, W.: Inequalities for $E k(X, Y)$ when the marginals are fixed. Z. Wahrscheinizchkeitstheorie verw. Gebiete $36,285-294(1976)$.

3. Das Gupta, S., Eaton, M.L., O1kin, I., Perlman, M., Savage, L.J., Sobe1, M.: Inequalities on the probability content of convex regions for elliptically contoured distributions. Proc. Sixth Berkeley Symp. Math. Statist. Prob. 2, 241-264. Berkeley: University of California Press, 1972.

4. Kamae, T., Krenge1, U., O'Brien, G.L.: Stochastic inequalities on partially ordered spaces. Ann. Probability 5, 899-912 (1977).

5. Kemperman, J.H.B.: On the FKG-inequality for measures on a partially ordered space. Proc. Akad. Wetenschappen, Ser. A. 80, 313-331 (1977).

6. Nevius, S.E., Proschan, F., Sethuraman, J.: Schur functions in statistics II. Stochastic majorization. Ann. Statist. 5, 263-273 (1977).

7. Roberts, A.W., Varberg, D.E.: Convex Functions. New York: Academic Press, 1973.

8. Schoenberg, I.J.: Metric spaces and completely monotone functions. Ann. Math. $39,811-841$ (1938).

9. Slepian, D.: The one-sided barrier problem for Gaussian noise. Belz System Tech. J. 41, 463-501 (1962):

10. Strassen, V.: The existence of probability measures with given marginals. Ann. Math. Statist. 36, 423-439 (1965).

11. Sudakov, V.N.: The existence of probability measures with specified projections. Math. Notes Acad. Sei. USSR 14, 886-888 (1973).

12. Tchen, A.H-T.: Inequalities for distributions with given marginals. Tech. Report No. 19, Dept. of Statistics, Stanford University (1976).

13. Widder, D.V.: The Laplace Transform. Princeton: Princeton University Press, 1946. 
UNCLASSIFIED

SECURITY CLASSIFICATION OF THIS PAGE (When Dete Entered),

\begin{tabular}{|c|c|}
\hline REPORT DOCUMENTATION PAGE & $\begin{array}{c}\text { READ INSTRUCTIONS } \\
\text { BEFORE COMPLETING FORM }\end{array}$ \\
\hline 2. GOVT ACCESSION NO. & 3. RECIPIENT'S CATALOG NUMBER \\
\hline \multirow[t]{2}{*}{$\begin{array}{l}\text { 4. TITLE (and Subutr) } \\
\text { Probability and Expectation Inequalities }\end{array}$} & $\begin{array}{l}\text { 5. TYPE OF REPONT Q PERIOD COVEREO } \\
\text { TECHNICAL }\end{array}$ \\
\hline & $\begin{array}{l}\text { 6. PERFORMING OTG. REPORT NUMAER } \\
\text { Mimeo Series No. } 1263\end{array}$ \\
\hline $\begin{array}{l}\text { 7. AUTHOR(0) } \\
\text { Stamatis Cambanis and Gordon Simons }\end{array}$ & $\begin{array}{l}\text { 8. CONTRACT OR GRANT NUMBER(B) } \\
\text { Grant AFOSR }=75-2796 \\
\text { Grant MCS78-01240 }\end{array}$ \\
\hline 9. PERFORMING ORGANIZATION NAME AND ADDRESS & $\begin{array}{l}\text { 10. PROGRAM ELEMENT PROJECT, TASK } \\
\text { AREA W WORK UNIT NUMEERS }\end{array}$ \\
\hline \multirow{2}{*}{$\begin{array}{l}\text { 11. CONTROLLING OFFICE NAME AND ADDRESS } \\
\text { Air Force Office of Scientific Research } \\
\text { Bolling Air Force Base } \\
\text { Washington, D.C. } 20332\end{array}$} & $\begin{array}{l}\text { 12. REPORT DATE } \\
\text { January } 1980\end{array}$ \\
\hline & $\begin{array}{l}\text { 13. NUMBEF OF PAGES } \\
49\end{array}$ \\
\hline \multirow[t]{2}{*}{ T7. MONITORING AGENCY NAME AODRESS(ll dilforont from Controllind Oflico) } & $\begin{array}{l}\text { 15. SECURITY CLASS. (of this teport) } \\
\text { UNCLASSIFIED }\end{array}$ \\
\hline & 15. DECLASSIFICATION/DOWNGRADING \\
\hline
\end{tabular}

Approved for Public Release: Distribution Unlimited

17. DISTFIBUTION STATEMENT (of the abetract on lored in Block 20, if difforent from Report)

10. SUPPLEMENTARY NOTES

19. KEY WORDS (Continue on reverse side If necessary and identlly by block number)

probability inequalities, expectation inequalities, normal distribution, elliptically contoured distributions, stochastic orderings, quasi-monotone functions, n-monotone functions

20. ABSTRACT (Continuo on reverdo alde If noceseary and identity by block number)

This paper introduces a mathematical framework within which a wide variety of known and new inequalities can be viewed from a common perspective. Probability and expectation inequalities of the following types are considered: (a) $P(Z \in A) \geq P\left(Z^{\prime} \in A\right)$ for some class of sets $A$, (b) $E k(Z) \geq E k\left(Z^{\prime}\right)$ for some class of functions $k$, and (c) $E l(Z) \geq E k\left(Z^{\prime}\right)$ for some class of pairs of functions $\ell$ and $k$. It is shown, sometimes using explicit constructions of $Z$ and $Z^{\prime}$, that, in several cases, (a) $\Leftrightarrow(b) \Leftrightarrow(c)$; included here are cases 
20. of normal and elliptically contoured distributions. A case where (a) $\Rightarrow$ (b) $\Leftrightarrow$ (c) is studied and is expressed in terms of "n-monotone" functions for (some of) which integral representations are obtained. Also, necessary and sufficient conditions for (c) are given. 